\title{
DESIGN EFFICIENCY AND OPTIMAL VALUES OF REPLICATED CENTRAL COMPOSITE DESIGNS WITH FULL FACTORIAL PORTIONS
}

\author{
Iwundu M.P. ${ }^{*}$ and Oko E.T. ${ }^{2}$ \\ ${ }^{1}$ Department of Mathematics and Statistics, University of Port Harcourt, Nigeria. \\ ${ }^{2}$ Department of Mathematics and Statistics, Rivers State University, Nigeria \\ *Corresponding author E-mail:mary.iwundu@uniport.edu.ng
}

Cite this article:

Iwundu M.P., Oko E.T. (2021), Design Efficiency and Optimal Values of Replicated Central Composite Designs with Full Factorial Portions. African Journal of Mathematics and Statistics Studies 4(3), 89-117. DOI: 10.52589/AJMSS-

AJWDYPOV.

\section{Manuscript History}

Received: 2 July 2021

Accepted: 3 Aug 2021

Published: 28 Oct 2021

Copyright $\odot 2020$ The Author(s). This is an Open Access article distributed under the terms of Creative Commons AttributionNonCommercial-NoDerivatives 4.0 International (CC BY-NC-ND 4.0 ), which permits anyone to share, use, reproduce and redistribute in any medium, provided the original author and source are credited.
ABSTRACT: Efficiency and optimal properties of four varieties of Central Composite Design, namely, SCCD, RCCD, OCCD and FCCD and having $r_{f}$ replicates of the full factorial portion, $r_{\alpha}$ replicates of the axial portion and $r_{c}$ replicates of the center portion are studied in four to six design variables. Optimal combination, $\left[r_{f}: r_{\alpha}: r_{c}\right]$ of design points associated with the three portions of each central composite design is presented. For SCCD, the optimal combinations resulting in $A$ - and D-efficient designs generally put emphasis on replicating the center portion of the SCCD. However, replicating the center and axial portions allows for G-optimal and efficient designs. For RCCD, the optimal combinations resulting in A-and D-efficient designs generally put emphasis on replicating the factorial and center portions of the RCCD. However, replicating the center and axial portions allows for $G$-optimal and efficient designs. For OCCD, the optimal combinations resulting in A-optimal and efficient designs generally put emphasis on replicating the axial and center portion of the OCCD. The optimal combinations resulting in $G$-optimal and efficient designs generally put emphasis on replicating the factorial and axial portions of the OCCD. To achieve designs that are D-optimal and D-efficient, the optimal combination of design points generally put emphasis on replicating the center portion of the OCCD. For FCCD, the optimal combinations of design points resulting in A-efficient designs put emphasis on replicating the axial portion of the FCCD. The optimal combinations resulting in $G$-optimal and efficient designs as well as G-optimal and efficient designs generally put emphasis on replicating the factorial and axial portions of the FCCD. It is interesting to note that for FCCD in five design variables, any $r^{\text {th }}$ complete replicate of the distinct design points of the combination $\left[r_{f}: r_{\alpha}: r_{c}\right]$ resulted in a D-efficient design. Many super-efficient designs having efficiency values greater than 1.0 emerged under the D-criterion. Unfortunately, these designs did not perform very well under $A$ - and G-criteria, having some efficiency values much below 0.5 or just about 0.6 .

KEYWORDS: SCCD, RCCD, OCCD, FCCD, Optimal Values, Design Efficiency 


\section{INTRODUCTION}

Full factorial experiment, refers to experiment with two or more factors, and each factor with discrete possible values or levels. The choice of the level of factors is determined by the experimenter to suit the type(s) of research he or she undertakes. The effect of each factor as well as the interaction effects between factors is carefully studied to ascertain how they influence the dependent or the response variable. Conventionally, the design is coded at low and high levels for each continuous factor as -1 and +1 respectively. The required number of experiments given $\mathrm{k}$ factors, is given by

for a non-replicated design

$$
N=2^{K-m}+2 k+n_{C}
$$

and

$$
N=2^{K-m} r_{f}+2 k r_{\alpha}+n_{C_{r}}
$$

for replicated design

where $\boldsymbol{r}_{\boldsymbol{f}}$ the number of replications of the factorial point is $\boldsymbol{r}_{\boldsymbol{\alpha}}$ is the number of replications of the axial point and $\boldsymbol{n}_{\boldsymbol{r} \boldsymbol{c}}$ is the number of replications of the center points

The factorial design is considered a first order model design which consists of constant, linear and interaction terms, and takes the form of

$$
y=\beta_{0}+\sum_{i=1}^{k} \beta_{i} x_{i}+\sum_{i=1}^{k-1} \sum_{i<j=2}^{k} \beta_{i j} x_{i} x_{j}
$$

Where $\mathrm{y}$ is measured response; $x_{i}=$ the coded independent variables; $i=1,2, \ldots, \mathrm{k} ; \beta$ 's are unknown parameters and $\varepsilon$ is the random error with mean zero and variance $\sigma^{2}$

When there are no replications in a two level factorial design which involves only continuous factor, the error sum of squares cannot be estimated because there is no degree of freedom available; hence, the model coefficients cannot be statistically tested. In this situation, replication becomes unavoidable. It is necessary to consider a second-order model for the purpose of obtaining a more precise estimate of experimental error and for modeling curvature. Potentially, a Second-Order polynomial model contains all the terms of the First-Order model, all quadratic terms and all cross product terms. It is expressed in the form.

$$
\begin{gathered}
y=\beta_{0}+\sum_{i=1}^{k} \beta_{i} x_{i}+\sum_{i=1}^{k-1} \sum_{j=i+1}^{k} \beta_{i j} x_{i} x_{j} \\
+\sum_{k=1}^{k} \beta_{i i} x_{i}^{2}+\varepsilon
\end{gathered}
$$


where $\mathrm{y}$ is measured response; $x_{i}=$ the coded independent variables; $i=1,2, \ldots, \mathrm{k} ; \beta$ 's are unknown parameters and $\varepsilon$ is the random error with mean zero and variance $\sigma^{2}$

In this research work, our focus is on one of the most flexible and widely used second-order design - the Central Composite Design. According to Box and Wilson (1951), Central Composite Design is the most popular class of second order designs. They are replications of $2^{\mathrm{k}}$ factorial points or $2^{\mathrm{k}-\mathrm{p}}$ fractional factorial points of resolution $\mathrm{V}$ with replications of the cube, the axial and the center portions. The various replications are denoted in this paper by $r_{f}, r_{\alpha}$ and $n_{r c}$ respectively.The CCD is constructed by first creating a $2^{\mathrm{k}}$ factorial or the $2^{k-p}$ fraction designs and then appending a set of extra runs referred to as axial or star point. The axial points supply the extra levels required to fit a second order model in all factors. The $2^{k}$ the $2 \mathrm{k}-\mathrm{p}$ portion of the design allows us to fit first order terms and interactions, the axial portion allows us to fit quadratic terms in the factors. So, typically, one first runs a factorial portion with center point, this lets us estimate main effects and two-way interactions, and the center point lets us test for curvature. Replication of the center runs allows us to generate a pure error.

In order to obtain a better estimate of all linear and product term coefficient, squared term coefficient and also to estimate pure error, it is necessary to repeat the cube, the axial and the center portions a few or several times, this is referred to as replication in Central Composite Design. Replication in the Central Composite Design may take the form of Complete or Partial replications.

In this work, equal and partial replications of the cube, the star and the center points are employed. Equal replication implies replicating the cube point, the axial point and the center point equally while partial replication involves replicating either the cube point or the axial point or the center point.

Our focus is to locate the optimum combination of replications of the different portions, i.e., the cube, the axial and the center portions that yield optimal designs. To achieve this, a total of twenty seven (27) equal and partial replicated random experiments for $\mathrm{k}=4,5$ and 6 for the various portions of the varying CCD's, namely, Spherical Central Composite Design (SCCD), Rotatable Central Composite Design (RCCD), Orthogonal Central Composite Design (OCCD) and Face Centered Central Composite Design (FCCD).

An appropriate experimental design is based on finding the best optimality criterion in which larger efficiency value implies a better design; Boonorm and Borkowski (2012).

The adoption of an appropriate experimental design capable of representing the response surface design greatly influences the efficiency of the experimental design Francis and Lilian (2018).

Many researchers have carried out different works on replication of Central Composite Designs. Chigbu and Ukaegbu (2017) examined earlier and later studies on the partial replication of the response surface central composite designs (CCDs). The results showed that the optimum performance of the replicated variations of the CCD depends on the axial distance, $\alpha$, and also the cuboidal or spherical design region when the factorial and axial points are replicated in both design regions studied, no particular replicated variation of the CCD is consistently optimum. They concluded that in most cases replicating the axial points, improves the designs. 
Ibanga, (2013) compared some variations of experimental points of central composite designs in the presence of complete replication under rotatable and orthogonal design restrictions using the A-, D- and E- optimality criteria The efficiency values obtained suggest that replicated cubes plus replicated star points are better than partial replication of cube and star points under the design restrictions of rotatability and orthogonally.

Iwundu (2015) carefully examined the optimal partially replicated cube, star and center runs in Face centered central composite designs. The cube points were replicated while holding the star points and center points were not replicated, the star points were replicated while the cube points and star points were not replicated and the center points were replicated while holding the cube points and the star points fixed. The efficiencies of the designs were assessed using the D and G optimality criteria. The results showed that with the Face centered central composite design replicating the cube portion twice with fixed star and center points performed better than other variations under D and G-optimality criteria. It was also observed that replicating the cube points was more efficient than replicating the center points, and as such, emphasis should shift from replication of only center points, as non-center points performed better.

Two variations of central composite designs under the orthogonal and rotatable restriction using the D optimality criterion were compared by Chigbu and Ohaegbulem (2011) and they concluded that the replicated cube plus one star variation is better than the replicated star plus one cube variation under both restrictions.

Nduka and Chigbu (2014) compared two variation of $\mathrm{N}$ point orthogonal and rotatable central Composite design based on Schur's ordering of design which says

$$
\sum_{i=1}^{k} \lambda_{i}(\xi) \geq \sum_{i=1}^{k} \lambda_{i}(\eta) ;(k=1,2, \ldots, p)
$$

as well as the D optimality and A optimality criteria. They came to the conclusion that by Schur's ordering of designs; a fixed axial point with replicated cube point is better than a fixed cube point plus replicated axial point. It was further demonstrated that the result remained the same for both A-optimality and D-Optimality.

Francis and Lilian (2018) investigated the effect of replicating the cube point, the axial point or center point and the results suggest that replication affects the different criteria in different dimensions because what improves one criterion positively may have negative impact on different criterion. It was further suggested that experimenters should be willing to sacrifice design efficiency to gain a pure error degree of freedom for lack of fit in the case of a decrease in efficiency of the replicated star or cube portion.

Iwundu (2017) studied the effects of addition of $n_{C}$ center points on the optimality of BoxBehnken and Box-Wilson second-order designs. Relationships were seen to exist between optimal design properties and varying size of the designs by the addition of center points, the relationships between the Box-Behnken designs and the central composite design defined at $\alpha$ 
$=\sqrt{k}$ and $\alpha=f^{\frac{1}{4}}$ are very strong and variations seem to exist with central composite designs defined at $\alpha=1$

\section{METHODOLOGY}

In this work, equal replication and partial replication for the various central composite designs; Spherical, Rotatable, Orthogonal, and Face centered central composite designs were employed to determine at what level of combination of the replication of the factorial point, the axial point and the center point, an optimum can be attained using the A, D and G optimality criteria. This was computed using some statistical Softwares, namely, MINITAB, DESIGN

EXPERT, MATLAB and JMP. This study covers the Central Composite Design with $\mathrm{k}=4,5$ and 6 design variables.

\section{Model and Design}

The Central composite design (CCD) emanated from the response surface designs and is the most popular and commonly used classes of experimental design for fitting a second-order response surface model and is given as

$$
y=\beta_{0}+\sum_{i=1}^{k} \beta_{i} x_{i}+\sum_{i=1}^{k-1} \sum_{j=i+1}^{k} \beta_{i j} x_{i} x_{j}+\sum_{k=1}^{k} \beta_{i i} x_{i}^{2}+\varepsilon
$$

where $\mathrm{y}$ is measured response; $x_{i}=$ the coded independent variables; $i=1,2, \ldots, \mathrm{k} ; \beta$ 's are unknown parameters and $\varepsilon$ is the random error with mean zero and variance $\sigma^{2}$

The Central Composite Design is made up of the cube portion, the axial or star portion and the center runs. The four varieties of the Central Composite Design discussed in this research paper are: Spherical Central Composite Design (SCCD), the Rotatable Central Composite Design (RCCD), the Orthogonal Central Composite Design (OCCD) and the Face Centered Central Composite Design (FCCD).

The Spherical Central Composite design is a design that puts all the factorial and axial design point on the surface of a sphere of radius $\sqrt{k}$, and one of the major features is rotatability.

Rotatable Central Composite Design is a design that has the same prediction variance for any defined point equidistant from the design center. The alpha value is calculated as

$$
\alpha=F^{\frac{1}{4}}
$$

where $\mathrm{F}$ is the number of factorial or fractional factorial points.

Orthogonal Central Composite Design plays an important role as a second-order design. A design is said to be orthogonal if the effect of any factor balances out (totals up to zero) across the effects of other factors or if the off-diagonal elements of the information matrix $X^{\prime} X$ are zero. As in Khuri (1996), the condition for making a CCD to be orthogonal is by setting 


$$
\alpha=\left[\frac{\sqrt{N f}-f}{2}\right]^{\frac{1}{2}}
$$

The Face Centered Cube Design shall also be explored. This type of design places the axial point at the center of each face of the factorial space. Setting $\alpha=1$ makes the CCD Face Centered Cube Design. For a given second-order model, an N x p model matrix shall be formed using the design and the model. For example, the model matrix associated with central composite design with no replications and in $\mathrm{k}$ design variables for axial distance $\alpha$ and design size, $\mathrm{N}$ is represented in Algebraic form as

$\mathrm{X}=$

\begin{tabular}{|ccccccccccccc}
1 & $x_{11}$ & $x_{12}$ & $\cdots$ & $x_{1 k}$ & $x_{11}^{2}$ & $x_{12}^{2}$ & $\cdots$ & $x_{1 k}^{2}$ & $x_{11} x_{12}$ & $x_{11} x_{13}$ & $\cdots$ & $x_{1(k-1)} x_{1 k}$ \\
1 & $x_{21}$ & $x_{22}$ & $\cdots$ & $x_{2 k}$ & $x_{21}^{2}$ & $x_{22}^{2}$ & $\cdots$ & $x_{2 k}^{2}$ & $x_{21} x_{22}$ & $x_{21} x_{23}$ & $\cdots$ & $x_{2(k-1)} x_{2 k}$ \\
1 & $x_{31}$ & $x_{32}$ & $\cdots$ & $x_{3 k}$ & $x_{31}^{2}$ & $x_{32}^{2}$ & $\cdots$ & $x_{3 k}^{2}$ & $x_{31} x_{32}$ & $x_{31} x_{33}$ & $\cdots$ & $x_{3(k-1)} x_{3 k}$ \\
$\vdots$ & $\vdots$ & $\vdots$ & $\vdots$ & $\vdots$ & $\vdots$ & $\vdots$ & $\vdots$ & $\vdots$ & $\vdots$ & $\vdots$ & $\vdots$ & $\vdots$ \\
1 & $x_{n 1}$ & $x_{n 2}$ & $\cdots$ & $x_{n k}$ & $x_{n 1}^{2}$ & $x_{n 2}^{2}$ & $\cdots$ & $x_{n k}^{2}$ & $x_{n 1} x_{n 2}$ & $x_{n 1} x_{n 3}$ & $\cdots$ & $x_{n(k-1)} x_{n k}$ \\
1 & $-\alpha$ & 0 & $\cdots$ & 0 & $\alpha^{2}$ & 0 & $\cdots$ & 0 & 0 & 0 & $\cdots$ & 0 \\
1 & $\alpha$ & 0 & $\cdots$ & 0 & $\alpha^{2}$ & 0 & $\cdots$ & 0 & 0 & 0 & $\cdots$ & 0 \\
1 & 0 & $-\alpha$ & $\cdots$ & 0 & 0 & $\alpha^{2}$ & $\cdots$ & 0 & 0 & 0 & $\cdots$ & 0 \\
1 & 0 & $\alpha$ & $\cdots$ & 0 & 0 & $\alpha^{2}$ & $\cdots$ & 0 & 0 & 0 & $\cdots$ & 0 \\
1 & 0 & 0 & $\cdots$ & $-\alpha$ & 0 & 0 & $\cdots$ & $\alpha^{2}$ & 0 & 0 & $\cdots$ & 0 \\
$\vdots$ & $\vdots$ & $\vdots$ & $\vdots$ & $\alpha$ & $\vdots$ & $\vdots$ & $\vdots$ & $\alpha^{2}$ & $\vdots$ & $\vdots$ & $\vdots$ & $\vdots$ \\
1 & 0 & 0 & $\cdots$ & 0 & 0 & 0 & $\cdots$ & 0 & 0 & 0 & $\cdots$ & 0
\end{tabular}

The Information Matrix denoted by $\mathbf{J}$ in this research work is given as

$$
\mathrm{J}=\mathrm{X}^{\mathrm{T}} \mathrm{X}
$$

The inverse of the information matrix denoted by $\mathrm{Z}$ in this research work is written as

$$
\mathrm{Z}=\left(\mathrm{X}^{\mathrm{T}} \mathrm{X}\right)^{-1}
$$


The Normalized Information Matrix otherwise known as the Moment Matrix denoted by $\mathrm{M}$ is given as

$$
\mathrm{M}=\frac{X^{T} X}{N}
$$

where $\mathrm{N}$ the total number of runs is used as a penalty for the larger design.

\section{Optimality Criteria}

In studying the design efficiencies of replicated central composite designs with full factorial portions, the A, D and $\mathrm{G}$ design efficiencies shall be employed to assess the quality of the designs.

According to Chernoff (1953), the A-Optimality criterion seeks to minimize the trace of the inverse of the information matrix $\left(X^{\prime} X\right)$. A-efficiency is directly related to minimizing the individual variances of the model parameters; it provides a way of comparing designs across different sample sizes.

\section{A-Optimality}

This criterion introduced by Chemoff (1953) seeks to minimize the trace of the inverse of the information matrix $\mathrm{X}^{\mathrm{T}} \mathrm{X}$, that is

$$
A_{\text {opt }}=\left\{\min \left[\operatorname{trace}\left(\mathrm{X}^{\mathrm{T}} \mathrm{X}\right)^{-1}\right]\right\}
$$

Where $\mathrm{X}$ is the design matrix and trace is the sum of the design elements of the matrix. The Aefficiency is given as

$$
A_{\text {eff }}=\frac{100 p}{\operatorname{trace}\left[N\left(X^{\prime} X\right)^{-1}\right]}
$$

\section{D-Optimality}

The D-optimality criterion was the first alphabetical optimality criterion established, according to Wald (1943), this is based on the determinant of $\mathrm{X}^{\mathrm{T}} \mathrm{X}$ which is inversely proportional to the square of the volume of the confidence region on the regression coefficients. It indicates how well the set of coefficients are estimated. Therefore, a smaller $\left|X^{T} X\right|$ or equivalently, a larger $\left|\left(X^{\mathrm{T}} \mathrm{X}\right)^{-1}\right|$ implies poorer estimation of the regression coefficient in the model. The goal of D-optimality is to maximize $\left|X^{T} X\right|$ or equivalently minimize $\left|\left(X^{T} X\right)^{-1}\right|$ where $X$ is the design matrix. Mathematically,

$$
D_{o p t}=\left[\max \left|\left(\mathrm{X}^{\mathrm{T}} \mathrm{X}\right)\right|\right] \text { or }\left[\min \left|\left(\mathrm{X}^{\mathrm{T}} \mathrm{X}\right)^{-1}\right|\right]
$$

The D-efficiency according to Crosier (1993) is the $\mathrm{p}^{\text {th }}$ root of the ratio of $\frac{\operatorname{det}\left(X^{\prime} X\right)}{N^{P}}$ to maximum possible value of $\frac{\operatorname{det}\left(X^{\prime} X\right)}{N^{P}}$ for any design defined on the same region. The D-efficiency is

$D_{\text {eff }}=100 \frac{\left|X^{\prime} X\right|^{\frac{1}{p}}}{N}$ 


\section{G-Optimality}

The aim of G-optimality criterion; is to minimize the maximum prediction variance in the design region. Hence,

$$
D_{o p t}=\min \left[N \hat{\sigma}_{\max }^{2}\right]
$$

The G-efficiency of a design is defined as

$$
\mathrm{G}=\frac{P}{V(x)_{\max }}
$$

Where $\mathrm{p}$ is the number of parameters in the model and $\mathrm{V}(\mathrm{x}) \max$ is maximum scaled variance of prediction. The variance of the function at $x$ according to Myer (1966) is

$$
\mathrm{V}(\hat{y}(\mathrm{x}))=\frac{V(x) \sigma^{2}}{N}
$$

where $\mathrm{V}(\mathrm{x})=\mathrm{N} \underline{\underline{x}}\left(X^{\prime} X\right)^{-1} \underline{\underline{x}}$ is the scaled prediction variance for any point $\underline{x}$ in the design region.

Thus

$\operatorname{Var}(\hat{y}(\mathrm{x}))=\underline{\underline{x}}^{\prime} M^{-1} \underline{\underline{x}}$.

The vector $\underline{\underline{x}}$ is the row vector of the design matrix $\mathrm{X}$, associated with the design point $\underline{x}$.

G-efficiency thus examines the maximum value of $\mathrm{V}(\underline{x})=\frac{N \operatorname{Var}(\hat{y}(x))}{\sigma^{2}}$ within the design region with respect to its theoretical minimum variance p. Therefore, A G-optimality and the corresponding G-efficiency emphasize the use of designs for which the maximum $\frac{N v a r[\hat{y}(x)]}{\sigma^{2}}$ in the region of the design is not too large.

\section{RESULTS}

In this research work, the optimal A, D and G-optimality values were obtained for equal and partial replication of cube, axial and center points using selected varieties of the Central Composite Designs; the SCCD, RCCD, OCCD and FCCD, for factor $k=4,5$ and 6. Various combinations of replications of the cube, the axial and the center portions were employed to track the exact points where optimal values occur as can be seen in the tables below. 
Table 1 Optimality properties for SCCD with Full Factorial Replicates in $k=4$ variables

\begin{tabular}{|c|c|c|c|c|c|c|c|c|c|c|c|c|c|}
\hline \multirow[b]{2}{*}{$\begin{array}{l}\text { Design } \\
\text { type: } \\
\text { SCCD }\end{array}$} & \multirow[b]{2}{*}{$\mathbf{N}$} & \multirow[b]{2}{*}{$\mathbf{P}$} & \multirow[b]{2}{*}{$\boldsymbol{\alpha}$} & \multirow[b]{2}{*}{$r_{f}$} & \multirow[b]{2}{*}{$r_{\alpha}$} & \multirow[b]{2}{*}{$n_{c}$} & \multirow[b]{2}{*}{ Aopt } & \multicolumn{2}{|c|}{ Dopt } & \multirow[b]{2}{*}{ Gopt } & \multirow[b]{2}{*}{$A e f$} & \multirow[b]{2}{*}{ Deff } & \multirow[b]{2}{*}{ Geff } \\
\hline & & & & & & & & $\max \mid\left(\frac{X^{\prime} X}{N}\right)$ & $\min \mid\left(\frac{X^{\prime} X}{N}\right)$ & & & & \\
\hline 1 & 25 & 15 & 2 & 1 & 1 & 1 & 47.40 & 0.0188 & 53.188 & 25.00 & 31.65 & 76.73 & 60.00 \\
\hline 2 & 26 & 15 & 2 & 1 & 1 & 2 & 33.05 & 0.0209 & 47.894 & 15.17 & 45.40 & 77.27 & 98.90 \\
\hline 3 & 27 & 15 & 2 & 4 & 1 & 3 & 28.70 & 0.0178 & 56.241 & 15.75 & 52.29 & 76.44 & 95.24 \\
\hline 4 & 33 & 15 & 2 & 1 & 2 & 1 & 59.67 & 0.0098 & 101.541 & 33.00 & 25.19 & 73.49 & 45.46 \\
\hline 5 & 34 & 15 & 2 & 1 & 2 & 2 & 40.12 & 0.0126 & 79.448 & 18.06 & 37.40 & 74.70 & 83.05 \\
\hline 6 & 35 & 15 & 2 & 1 & 2 & 3 & 33.99 & 0.0122 & 81.814 & 18.59 & 44.12 & 74.56 & 80.67 \\
\hline 7 & 41 & 15 & 2 & 1 & 3 & 1 & 72.28 & 039 & 255.779 & 41.00 & 20.76 & 69.10 & 36.59 \\
\hline 8 & 42 & 15 & 2 & 1 & 3 & 2 & 47.80 & 0.0054 & 183.576 & 21.00 & 31.40 & 70.65 & 71.43 \\
\hline 9 & 43 & 15 & 2 & & 3 & 3 & & 0.0057 & 174.186 & 21.50 & 37.54 & 70.89 & 69.77 \\
\hline 10 & 41 & 15 & 2 & & 1 & 1 & 67.16 & 0.0093 & 107.907 & 36.59 & 22.34 & 73.19 & 36.59 \\
\hline 11 & 42 & 15 & 2 & 2 & 1 & 2 & 42.55 & 0.0129 & 77.446 & 21.00 & 35.27 & 74.83 & 71.43 \\
\hline 12 & 43 & 15 & 2 & 2 & 1 & 3 & 34.57 & 0.0136 & 73.485 & 21.50 & 43.38 & 75.09 & 69.77 \\
\hline 13 & 49 & 15 & 2 & 2 & 2 & 1 & 77.08 & 0.0127 & 78.566 & 49.00 & 19.46 & 74.76 & 530.61 \\
\hline 14 & 50 & 15 & 2 & & 2 & 2 & & & 53.188 & 25.00 & 31.65 & 76.73 & 60.00 \\
\hline 15 & 51 & 15 & 2 & 2 & 2 & 3 & 37.74 & 0.0210 & 47.723 & 17.00 & 39.77 & 77.28 & 88.24 \\
\hline 16 & 57 & 15 & 2 & & 3 & 1 & 88.07 & 096 & 104.090 & 57.00 & 17.04 & 73.37 & 26.32 \\
\hline 17 & 58 & 15 & 2 & 2 & 3 & 2 & & & 67.558 & 9.00 & 28.12 & 75.52 & 51.72 \\
\hline 18 & 59 & 15 & 2 & & 3 & 3 & 41.95 & 0.0172 & 58.203 & 19.67 & 35.74 & 76.27 & 76.27 \\
\hline 19 & 57 & 15 & 2 & 3 & 1 & 1 & & 041 & 246.732 & 57.00 & 17.04 & 69.27 & 26.32 \\
\hline 20 & 58 & 15 & 2 & 3 & 1 & 2 & 53.36 & 0.0062 & 160.137 & 29.00 & 28.12 & 71.29 & 51.72 \\
\hline 21 & 59 & 15 & 2 & 3 & 1 & 3 & 41.95 & 0.0072 & 137.963 & 27.39 & 35.74 & 72.00 & 54.76 \\
\hline 22 & 65 & 15 & 2 & 3 & 2 & 1 & 96.72 & 0.0088 & 113.437 & 65.00 & 15.51 & 72.95 & 23.08 \\
\hline 23 & 66 & 15 & 2 & 5 & 2 & 2 & 56.96 & 0.0140 & 71.315 & 33.00 & 26.33 & 75.24 & 45.46 \\
\hline 24 & 67 & 15 & 2 & 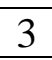 & 2 & 3 & 43.89 & 0.0168 & 59.574 & 51.72 & 34.18 & 76.15 & 51.72 \\
\hline 25 & 73 & 15 & 2 & 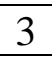 & 3 & 1 & 107.0 & 0.0151 & 66.029 & 73.00 & 14.02 & 73.26 & 20.55 \\
\hline 26 & 74 & 15 & 2 & 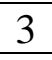 & 3 & 2 & 62.16 & 0.0153 & 65.232 & 37.00 & 24.12 & 75.69 & 40.54 \\
\hline 27 & 75 & 15 & 2 & 3 & 3 & 3 & 47.40 & 0.0188 & 53.188 & 25.00 & 31.61 & 76.73 & 60.00 \\
\hline
\end{tabular}


Table 2 Optimality properties for SCCD with Full Factorial Replicates in $k=5$ variables

\begin{tabular}{|c|c|c|c|c|c|c|c|c|c|c|c|c|c|}
\hline \multirow[b]{2}{*}{$\begin{array}{l}\text { Design } \\
\text { type: } \\
\text { SCCD } \\
\end{array}$} & \multirow[b]{2}{*}{$\mathbf{N}$} & \multirow[b]{2}{*}{$\mathbf{P}$} & \multirow[b]{2}{*}{$\alpha$} & \multirow[b]{2}{*}{$r_{f}$} & \multirow[b]{2}{*}{$r_{\alpha}$} & \multirow[b]{2}{*}{$n_{c}$} & \multirow[b]{2}{*}{ Aopt } & \multicolumn{2}{|c|}{ Dopt } & \multirow[b]{2}{*}{ Gopt } & \multirow[b]{2}{*}{ Aeff } & \multirow[b]{2}{*}{ Deff } & \multirow[b]{2}{*}{ Geff } \\
\hline & & & & & & & & $\max \mid\left(\frac{X^{\prime} X}{N}\right)$ & $\min \mid\left(\frac{X^{\prime} X}{N}\right)^{-1}$ & & & & \\
\hline 1 & 43 & 21 & 2.236 & 1 & 1 & 1 & 73.79 & 0.0096 & 104.00 & 43.00 & 28.45 & 80.16 & 48.84 \\
\hline 2 & 44 & 21 & 2.236 & 1 & 1 & 2 & 49.10 & 0.0119 & 84.28 & 23.89 & 42.75 & 80.96 & 87.92 \\
\hline 3 & 45 & 21 & 2.236 & 1 & 1 & 3 & 41.22 & 0.0111 & 90.06 & 24.43 & 50.93 & 80.71 & 85.97 \\
\hline 4 & 53 & 21 & 2.236 & 1 & 2 & 1 & 87.61 & 0.0069 & 145.67 & 53.00 & 23.98 & 78.88 & 39.62 \\
\hline 5 & 54 & 21 & 2.236 & 1 & 2 & 2 & 56.86 & 0.0093 & 107.85 & 27.00 & 36.95 & 80.02 & 77.78 \\
\hline 6 & 55 & 21 & 2.236 & 1 & 2 & 3 & 46.92 & 0.0095 & 105.90 & 23.53 & 44.79 & 80.10 & 89.23 \\
\hline 7 & 63 & 21 & 2.236 & 1 & 3 & 1 & 102.3 & 0.0026 & 377.60 & 63.00 & 20.54 & 75.38 & 33.33 \\
\hline 8 & 64 & 21 & 2.236 & 1 & 3 & 2 & 65.47 & 0.0038 & 262.80 & 32.00 & 32.07 & 76.70 & 65.63 \\
\hline 9 & 65 & 21 & 2.236 & 1 & 3 & 3 & 53.50 & 0.0041 & 242.63 & 26.60 & 39.25 & 76.99 & 78.94 \\
\hline 10 & 75 & 21 & 2.236 & 2 & 1 & 1 & 113.0 & 0.0025 & 402.04 & 75.00 & 18.59 & 75.16 & 28.00 \\
\hline 11 & 76 & 21 & 2.236 & 2 & 1 & 2 & 68.93 & 0.0038 & 265.48 & 38.00 & 30.48 & 76.66 & 55.26 \\
\hline 12 & 77 & 21 & 2.236 & 2 & 1 & 3 & 54.44 & 0.0043 & 232.90 & 37.04 & 38.60 & 77.14 & 56.69 \\
\hline 13 & 85 & 21 & 2.236 & 2 & 2 & 1 & 123.9 & 0.0061 & 162.70 & 85.00 & 16.94 & 78.47 & 24.71 \\
\hline 14 & 86 & 21 & 2.236 & 2 & 2 & 2 & 73.79 & 0.0096 & 104.00 & 43.00 & 28.46 & 80.16 & 48.84 \\
\hline 15 & 87 & 21 & 2.236 & 2 & 2 & 3 & 57.25 & 0.0113 & 88.38 & 29.00 & 36.68 & 80.78 & \begin{tabular}{|l|}
72.41 \\
\end{tabular} \\
\hline 16 & 95 & 21 & 2.236 & 2 & 3 & 1 & 136.6 & 0.0059 & 169.18 & 95.00 & 15.37 & \begin{tabular}{|l|l|}
78.32 \\
\end{tabular} & 22.11 \\
\hline 17 & 96 & 21 & 2.236 & 2 & 3 & 2 & 80.45 & 0.0095 & 105.39 & 48.00 & 26.10 & 80.11 & 43.75 \\
\hline 18 & 97 & 21 & 2.236 & 2 & 3 & 3 & 61.91 & 0.0114 & 87.35 & 32.33 & 33.92 & 80.83 & 64.95 \\
\hline 19 & 107 & 21 & 2.236 & 3 & 1 & 1 & 153.3 & 0.0007 & 1405 & 107.0 & 13.69 & 70.81 & 19.63 \\
\hline 20 & 108 & 21 & 2.236 & 3 & 1 & 2 & 89.96 & 0.0012 & 854.05 & 54.00 & 23.34 & 72.51 & 38.89 \\
\hline 21 & 109 & 21 & 2.236 & 3 & 1 & 3 & 69.02 & 0.0014 & 690.96 & 36.33 & 30.43 & 73.25 & \begin{tabular}{|l}
49.77 \\
\end{tabular} \\
\hline 22 & 117 & 21 & 2.236 & 3 & 2 & 1 & 162.5 & 0.0030 & 333.81 & 117.0 & 12.92 & 75.83 & 17.95 \\
\hline 23 & 118 & 21 & 2.236 & 3 & 2 & 2 & 93.10 & 0.0050 & 199.57 & 59.00 & 22.56 & 77.71 & 35.59 \\
\hline 24 & 119 & 21 & 2.236 & 3 & 2 & 3 & 70.09 & 0.0063 & 158.84 & 39.67 & 29.96 & 78.56 & 52.94 \\
\hline 25 & 127 & 21 & 2.236 & 3 & 3 & 1 & 174.2 & 0.0045 & 224.72 & 127.0 & 12.05 & \begin{tabular}{|l|}
77.27 \\
\end{tabular} & \begin{tabular}{|l|}
16.54 \\
\end{tabular} \\
\hline 26 & 128 & 21 & 2.236 & 3 & 3 & 2 & 98.82 & 0.0075 & 132.47 & 64.00 & 21.25 & 79.24 & 32.81 \\
\hline 27 & 129 & 21 & 2.236 & 3 & 3 & 3 & 73.79 & 0.0096 & 104.00 & 43.00 & 28.46 & 80.16 & 48.84 \\
\hline
\end{tabular}


Table 3 Optimality properties for SCCD with Full Factorial Replicates in $k=6$ variables

\begin{tabular}{|c|c|c|c|c|c|c|c|c|c|c|c|c|c|}
\hline \multirow[b]{2}{*}{$\begin{array}{l}\text { Design } \\
\text { type: } \\
\text { SCCD }\end{array}$} & \multirow[b]{2}{*}{$\mathbf{N}$} & \multirow[b]{2}{*}{$\mathbf{P}$} & \multirow[b]{2}{*}{$\boldsymbol{\alpha}$} & \multirow[b]{2}{*}{$r_{f}$} & \multirow[b]{2}{*}{$r_{\alpha}$} & \multirow[b]{2}{*}{$n_{c}$} & \multirow[b]{2}{*}{ Aopt } & \multicolumn{2}{|c|}{ Dopt } & \multirow[b]{2}{*}{ Gopt } & \multirow[b]{2}{*}{ Aeff } & \multirow[b]{2}{*}{ Deff } & \multirow[b]{2}{*}{ Geff } \\
\hline & & & & & & & & $\frac{\max \mid}{\left(\frac{X^{\prime} X}{N}\right) \mid}$ & $\min \mid\left(\frac{X^{\prime} X}{N}\right)^{-}$ & & & & \\
\hline 1 & 77 & 28 & 2.450 & 1 & 1 & 1 & 119.5 & 0.0032 & 315.04 & 77.00 & 23.44 & 81.43 & 36.36 \\
\hline 2 & 78 & 28 & 2.450 & 1 & 1 & 2 & 75.52 & 0.0044 & 226.07 & 39.69 & 37.07 & 82.40 & 70.55 \\
\hline 3 & 79 & 28 & 2.450 & 1 & 1 & 3 & 61.13 & 0.0046 & 215.31 & 40.20 & 45.80 & 82.54 & 69.66 \\
\hline 4 & 89 & 28 & 2.450 & 1 & 2 & 1 & 134.0 & 0.0049 & 203.50 & 89.00 & 20.89 & 82.71 & 31.46 \\
\hline 5 & 90 & 28 & 2.450 & 1 & 2 & 2 & 83.03 & 0.0072 & 139.10 & 45.00 & 33.73 & 83.84 & 62.22 \\
\hline 6 & 91 & 28 & 2.450 & 1 & 2 & 3 & 66.25 & 0.0079 & 126.39 & 30.33 & 42.26 & 84.13 & 92.31 \\
\hline 7 & 101 & 28 & 2.450 & 1 & 3 & 1 & 150.0 & 0.0026 & 378.00 & 101.0 & 18.66 & 80.90 & 27.73 \\
\hline 8 & 102 & 28 & 2.450 & 1 & 3 & 2 & 92.06 & 0.0040 & 249.04 & 51.00 & 30.42 & 82.11 & 54.90 \\
\hline 9 & 103 & 28 & 2.450 & 1 & 3 & 3 & 72.93 & 0.0046 & 218.18 & 34.33 & 38.39 & 82.50 & 81.55 \\
\hline 10 & 141 & 28 & 2.450 & 2 & 1 & 1 & 197.0 & 0.0003 & 3035.00 & 141.0 & 14.21 & 75.10 & 19.86 \\
\hline 11 & 142 & 28 & 2.450 & 2 & 1 & 2 & 115.6 & 0.00054 & 1850.00 & 71.00 & 24.22 & 76.44 & 39.44 \\
\hline 12 & 143 & 28 & 2.450 & 2 & 1 & 3 & 88.50 & 0.00067 & 11501.00 & 66.74 & 31.60 & 77.01 & 41.96 \\
\hline 13 & 153 & 28 & 2.450 & 2 & 2 & 1 & 208.0 & 0.0019 & 525.01 & 153.0 & 13.47 & 79.96 & 18.30 \\
\hline 14 & 154 & 28 & 2.450 & 2 & 2 & 2 & 119.5 & 0.0032 & 315.04 & 77.00 & 23.44 & 81.43 & 36.37 \\
\hline 15 & 155 & 28 & 2.450 & 2 & 2 & 3 & 90.11 & 0.0040 & 251.75 & 51.67 & 31.07 & 82.08 & 54.19 \\
\hline 16 & 165 & 28 & 2.450 & 2 & 3 & 1 & 221.9 & 0.0030 & 336.38 & 165.0 & 12.62 & 81.24 & 16.97 \\
\hline 17 & 166 & 28 & 2.450 & 2 & 3 & 2 & 126.4 & 0.0050 & 199.20 & 83.00 & 22.16 & 82.77 & 33.74 \\
\hline 18 & 167 & 28 & 2.450 & 2 & 3 & 3 & 94.66 & 0.0064 & 157.12 & 55.67 & 29.58 & 83.48 & 50.30 \\
\hline 19 & 205 & 28 & 2.450 & 3 & 1 & 1 & 275.6 & 0.000057 & 17673.00 & 205.0 & 10.16 & 70.52 & 13.66 \\
\hline 20 & 206 & 28 & 2.450 & 3 & 1 & 2 & 156.8 & 0.000099 & 10126.00 & 103.0 & 17.86 & 71.94 & 27.19 \\
\hline 21 & 207 & 28 & 2.450 & 3 & 1 & 3 & 117.3 & 0.00013 .00 & 7731.00 & 93.00 & 23.87 & 72.63 & 29.99 \\
\hline 22 & 217 & 28 & 2.450 & 3 & 2 & 1 & 283.9 & 0.00055 & 1820.00 & 217.0 & 9.864 & 76.48 & 12.90 \\
\hline 23 & 218 & 28 & 2.450 & 3 & 2 & 2 & 158.0 & 0.00097 & 1035.00 & 109.0 & 17.72 & 78.04 & 25.69 \\
\hline 24 & 219 & 28 & 2.450 & 3 & 2 & 3 & 116.1 & 0.0013 & 784.40 & 73.00 & 24.11 & 78.82 & 38.36 \\
\hline 25 & 229 & 28 & 2.450 & 3 & 3 & 1 & 296.6 & 0.0013 & 740.87 & 229.0 & 9.442 & 78.98 & 12.23 \\
\hline 26 & 230 & 28 & 2.450 & 3 & 3 & 2 & 163.7 & 0.0024 & 418.50 & 115.0 & 17.11 & 80.61 & 24.35 \\
\hline 27 & 231 & 28 & 2.450 & 3 & 3 & 3 & 119.5 & 0.0032 & 315.04 & 77.00 & 23.44 & 81.43 & 36.36 \\
\hline
\end{tabular}


Table 4 Optimality properties for RCCD with Full Factorial Replicates in $k=4$ variables

\begin{tabular}{|c|c|c|c|c|c|c|c|c|c|c|c|c|c|}
\hline \multirow[b]{2}{*}{$\begin{array}{l}\text { Design } \\
\text { type: } \\
\text { RCCD }\end{array}$} & \multirow[b]{2}{*}{$\mathbf{N}$} & \multirow[b]{2}{*}{$\mathbf{P}$} & \multirow[b]{2}{*}{$\alpha$} & \multirow[b]{2}{*}{$r_{f}$} & \multirow[b]{2}{*}{$r_{\alpha}$} & \multirow[b]{2}{*}{$n_{c}$} & \multirow[b]{2}{*}{ Aopt } & \multicolumn{2}{|c|}{ Dopt } & \multirow[b]{2}{*}{ Gopt } & \multirow[b]{2}{*}{ Aeff } & \multirow[b]{2}{*}{ Deff } & \multirow[b]{2}{*}{ Geff } \\
\hline & & & & & & & & $\max \mid\left(\frac{X^{\prime} X}{N}\right)$ & $\min \mid\left(\frac{X^{\prime} X}{N}\right)$ & & & & \\
\hline 1 & 25 & 15 & .000 & 1 & 1 & 1 & 47.40 & 0.0188 & 53.188 & 25.00 & 31.65 & 76.73 & 60.00 \\
\hline 2 & 26 & 15 & 2.000 & 1 & 1 & 2 & 33.05 & 0.0209 & 47.894 & 15.17 & 45.40 & \begin{tabular}{|l|}
77.27 \\
\end{tabular} & 98.90 \\
\hline 3 & 27 & 15 & 000 & 1 & 1 & 3 & 28.70 & 0.0178 & 56.241 & 15.75 & 52.29 & 76.44 & 95.24 \\
\hline 4 & 33 & 15 & 1.682 & 1 & 2 & 1 & 43.47 & $9.40 \times 10^{-4}$ & $1.064 \times 10^{3}$ & 18.91 & 34.52 & 62.83 & 79.32 \\
\hline 5 & 34 & 15 & 1.682 & 1 & 2 & 2 & 36.72 & $9.144 \times 10^{-4}$ & $1.094 \times 10^{3}$ & 19.37 & 40.86 & \begin{tabular}{|l|}
62.71 \\
\end{tabular} & 77.45 \\
\hline 6 & 35 & 15 & 682 & 1 & 2 & 3 & 33.73 & $7.951 \times 10^{-4}$ & $1.258 \times 10^{3}$ & 19.88 & 44.47 & 62.13 & 75.46 \\
\hline 7 & 41 & 15 & 520 & 1 & 3 & 1 & 39.89 & $1.043 \times 10^{-4}$ & $9.587 \times 10^{3}$ & 23.21 & 37.62 & 54.26 & 64.63 \\
\hline 8 & 42 & 15 & 1.520 & 1 & 3 & 2 & 37.75 & $9.153 \times 10^{-5}$ & $1.093 \times 10^{4}$ & 23.64 & 39.74 & \begin{tabular}{|l|}
53.79 \\
\end{tabular} & 63.45 \\
\hline 9 & 43 & 15 & 1.520 & 1 & 3 & 3 & 36.55 & $7.756 \times 10^{-5}$ & $1.289 \times 10^{4}$ & 24.12 & 41.05 & 53.20 & 62.20 \\
\hline 10 & 41 & 15 & 378 & 2 & 1 & 1 & 39.40 & 0.2334 & 4.2850 & 24.07 & 38.09 & 90.78 & 62.32 \\
\hline 11 & 42 & 15 & 378 & 3 & 1 & 2 & 31.30 & 0.2476 & 4.0393 & 24.08 & 47.95 & 91.13 & 62.28 \\
\hline 12 & 43 & 5 & 378 & 2 & 1 & 3 & 27.50 & 37 & 4.2799 & 24.37 & 54.56 & 90.78 & 61.55 \\
\hline 13 & 49 & 15 & 2.000 & 2 & 2 & 1 & 77.08 & 0.0127 & 78.566 & 49.00 & 19.46 & 74.76 & 30.61 \\
\hline 14 & 50 & 15 & 2.000 & 2 & 2 & 2 & 47.25 & 188 & 53.188 & 25.00 & 31.65 & 76.73 & 60.00 \\
\hline 15 & 51 & 15 & 000 & 2 & 2 & 3 & 37.74 & 210 & 47.723 & 17.00 & 39.77 & 77.28 & 88.24 \\
\hline 16 & 57 & 15 & 1.807 & 8 & 3 & 1 & 65.79 & 027 & 370.015 & 37.04 & 22.79 & 67.42 & 40.50 \\
\hline 17 & 58 & 15 & 1.807 & 8 & 3 & 2 & 47.83 & 34 & 291.203 & 22.85 & 31.36 & 68.51 & 65.66 \\
\hline 18 & 59 & 15 & 1.807 & 2 & 3 & 3 & 40.19 & 0.0037 & 270.013 & 16.96 & 37.32 & 68.86 & 88.45 \\
\hline 19 & 57 & 15 & 2.632 & 8 & 1 & 1 & 30.30 & 0.8071 & 1.2390 & 33.65 & 49.52 & 98.59 & 44.57 \\
\hline 20 & 58 & 15 & 2.632 & 3 & 1 & 2 & 27.16 & 0.7830 & 1.2772 & 33.51 & 55.24 & 98.39 & 44.77 \\
\hline 21 & 59 & 15 & 2.632 & 5 & 1 & 3 & 25.17 & 0.7306 & 1.3687 & 33.59 & 59.60 & 97.94 & 44.66 \\
\hline 22 & 65 & 15 & 2.213 & 3 & 2 & 1 & 65.84 & 0.0639 & 15.6610 & 42.24 & 22.81 & 83.27 & 35.51 \\
\hline 23 & 66 & 15 & 2.213 & 3 & 2 & 2 & 46.20 & 0.0838 & 11.9296 & 26.00 & 32.49 & 84.79 & 57.70 \\
\hline 24 & 67 & 15 & 2.213 & 3 & 2 & 3 & 37.79 & 0.0933 & 10.7219 & 19.24 & 37.71 & 85.39 & 77.97 \\
\hline 25 & 73 & 15 & 2.000 & 3 & 3 & 1 & 107.0 & 0.0151 & 66.029 & 73.00 & 14.02 & 73.26 & 20.55 \\
\hline 26 & 74 & 15 & 2.000 & 3 & 3 & 2 & 62.16 & 0.0153 & 65.232 & 37.00 & 24.12 & 75.69 & 40.54 \\
\hline 27 & 75 & 15 & 2.000 & 3 & 3 & 3 & 47.40 & 0.0188 & 53.188 & 25.00 & 31.61 & 76.73 & 60.00 \\
\hline
\end{tabular}


Table 5 Optimality properties for RCCD with Full Factorial Replicates in $k=5$ variables

\begin{tabular}{|c|c|c|c|c|c|c|c|c|c|c|c|c|c|}
\hline \multirow[b]{2}{*}{$\begin{array}{l}\text { Design } \\
\text { type: } \\
\text { RCCD }\end{array}$} & \multirow[b]{2}{*}{$\mathbf{N}$} & \multirow[b]{2}{*}{$\mathbf{P}$} & \multirow[b]{2}{*}{$\alpha$} & \multirow[b]{2}{*}{$r_{f}$} & \multirow[b]{2}{*}{$r_{\alpha}$} & \multirow[b]{2}{*}{$n_{c}$} & \multirow[b]{2}{*}{ Aopt } & \multicolumn{2}{|c|}{ Dopt } & \multirow[b]{2}{*}{ Gopt } & \multirow[b]{2}{*}{ Aeff } & \multirow[b]{2}{*}{ Deff } & \multirow[b]{2}{*}{ Geff } \\
\hline & & & & & & & & $\max \left|\left(\frac{X^{\prime} X}{N}\right)\right|$ & $\min \mid\left(\frac{X^{\prime} X}{N}\right)$ & & & & \\
\hline 1 & 43 & 21 & 2.378 & 1 & 1 & 1 & 66.72 & 0.0361 & 27.7216 & 38.28 & 31.48 & 85.37 & 54.86 \\
\hline 2 & 44 & 21 & 2.378 & 1 & 1 & 2 & 46.38 & 0.0421 & 23.7663 & 24.79 & 45.29 & 86.00 & 84.73 \\
\hline 3 & 45 & 21 & 2.378 & 1 & 1 & 3 & 39.37 & 0.0386 & 25.9008 & 25.90 & 53.34 & 85.65 & 83.04 \\
\hline 4 & 53 & 21 & 2.000 & 1 & 2 & 1 & 67.10 & 0.0010 & 956.8079 & 33.73 & 31.30 & 72.12 & 62.26 \\
\hline 5 & 54 & 21 & 2.000 & 1 & 2 & 2 & 51.94 & 0.0012 & 865.8060 & 23.81 & 40.43 & 72.46 & 88.19 \\
\hline 6 & 55 & 21 & 2.000 & 1 & 2 & 3 & 45.55 & 0.0011 & 916.4328 & 24.22 & 46.12 & 72.27 & 86.70 \\
\hline 7 & 63 & 21 & 1.807 & 1 & 3 & 1 & 52.28 & $9.041 \times 10^{-5}$ & $1.106 \times 10^{4}$ & 27.60 & 40.16 & 64.19 & 76.08 \\
\hline 8 & 64 & 21 & 1.807 & 1 & 3 & 2 & 48.17 & $8.310 \times 10^{-5}$ & $1.203 \times 10^{4}$ & 27.95 & 43.59 & 63.93 & 75.13 \\
\hline 9 & 65 & 21 & 1.807 & 1 & 3 & 3 & 45.71 & $7.311 \times 10^{-5}$ & $1.368 \times 10^{4}$ & 28.33 & 45.94 & 63.55 & 74.13 \\
\hline 10 & 75 & 21 & 2.828 & 2 & 1 & 1 & 43.29 & 0.6795 & 1.4716 & 43.50 & 48.54 & 98.19 & 48.28 \\
\hline 11 & 76 & 21 & 2.828 & 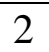 & 1 & 2 & 38.46 & 0.6588 & 1.5180 & 43.23 & 54.62 & 98.05 & 48.58 \\
\hline 12 & 77 & 21 & 2.828 & 2 & 1 & 3 & 35.45 & 22 & 1.6388 & 43.24 & 59.24 & 97.69 & 48.57 \\
\hline 13 & 85 & 21 & 2.378 & 2 & 2 & 1 & 102.1 & 0255 & 39.2542 & 68.19 & 20.59 & 83.98 & 30.80 \\
\hline 14 & 86 & 21 & 2.378 & 2 & 2 & 2 & 66.75 & 0359 & 27.8309 & 38.28 & 31.48 & 85.37 & 54.86 \\
\hline 15 & 87 & 21 & 2.378 & 2 & 2 & 3 & 53.35 & 0407 & 24.5456 & 26.80 & 39.37 & 85.88 & 78.36 \\
\hline 16 & 95 & 21 & 2.149 & 2 & 3 & 1 & 125.4 & 0030 & 336.9478 & 84.47 & 16.75 & 75.80 & 24.86 \\
\hline 17 & 96 & 21 & 2.149 & 2 & 3 & 2 & 78.09 & 045 & 222.2533 & 45.18 & 26.89 & 77.31 & 46.48 \\
\hline 18 & 97 & 21 & 2.149 & 2 & 3 & 3 & 61.24 & 0053 & 187.8732 & 31.04 & 34.28 & 77.93 & 67.65 \\
\hline 19 & 107 & 21 & 3.130 & 2 & 1 & 1 & 34.38 & 2902 & 0.4366 & 61.56 & 61.10 & 104.03 & 34.11 \\
\hline 20 & 108 & 21 & 3.130 & 3 & 1 & 2 & 32.79 & 2.1325 & 0.4689 & 61.36 & 64.05 & 103.68 & 34.23 \\
\hline 21 & 109 & 21 & 3.130 & 3 & 1 & 3 & 31.57 & 1.9621 & 0.5096 & 61.30 & 66.53 & 103.27 & 34.26 \\
\hline 22 & 117 & 21 & 2.632 & 3 & 2 & 1 & 63.34 & 0.2030 & 4.9255 & 37.44 & 31.17 & 92.70 & 56.09 \\
\hline 23 & 118 & 21 & 2.632 & 3 & 2 & 2 & 53.17 & 0.2242 & 4.4609 & 34.07 & 39.51 & 93.14 & 61.65 \\
\hline 24 & 119 & 21 & 2.632 & 3 & 2 & 3 & 46.63 & 0.2785 & 3.5902 & 33.93 & 44.67 & 93.31 & 61.90 \\
\hline 25 & 127 & 21 & 2.378 & 3 & 3 & 1 & 131.2 & 0.0203 & 49.3593 & 92.72 & 16.03 & 83.07 & 22.65 \\
\hline 26 & 128 & 21 & 2.378 & 3 & 3 & 2 & 85.29 & 0.0297 & 33.6172 & 54.01 & 24.64 & 84.61 & 38.88 \\
\hline 27 & 129 & 21 & 2.378 & 3 & 3 & 3 & 66.75 & 0.0359 & 27.8309 & 38.28 & 31.48 & 85.37 & 54.86 \\
\hline
\end{tabular}


Table 6 Optimality properties for RCCD with Full Factorial Replicates in $k=6$ variables

\begin{tabular}{|c|c|c|c|c|c|c|c|c|c|c|c|c|c|}
\hline \multirow{2}{*}{$\begin{array}{c}\text { Design } \\
\text { type: } \\
\text { RCCD }\end{array}$} & \multirow[b]{2}{*}{$\mathbf{N}$} & \multirow[b]{2}{*}{$\mathbf{P}$} & \multirow[b]{2}{*}{$\alpha$} & \multirow[b]{2}{*}{$r_{f}$} & \multirow[b]{2}{*}{$r_{\alpha}$} & \multirow[b]{2}{*}{$n_{c}$} & \multirow[b]{2}{*}{ Aopt } & \multicolumn{2}{|c|}{ Dopt } & \multirow[b]{2}{*}{ Gopt } & \multirow[b]{2}{*}{ Aeff } & \multirow[b]{2}{*}{ Deff } & \multirow[b]{2}{*}{ Geff } \\
\hline & & & & & & & & $\max \left|\left(\frac{X^{\prime} X}{N}\right)\right|$ & $\min \mid\left(\frac{X^{\prime} X}{N}\right)$ & & & & \\
\hline 1 & 77 & 28 & 2.828 & 1 & 1 & 1 & 71.17 & 0.1713 & 5.8368 & 43.79 & 39.37 & 93.91 & 3.94 \\
\hline 2 & 78 & 28 & 2.828 & 1 & 1 & 2 & 57.16 & 0.1791 & 5.5825 & 43.55 & 49.00 & 94.06 & 64.30 \\
\hline 3 & 79 & 28 & 2.828 & 1 & 1 & 3 & 50.34 & 0.1672 & 5.9803 & 43.70 & 55.64 & 93.83 & 4.08 \\
\hline 4 & 89 & 28 & 2.378 & 1 & 2 & 1 & 129.1 & 0.0025 & 394.1553 & 84.05 & 21.68 & 80.79 & 33.32 \\
\hline 5 & 90 & 28 & 2.378 & 1 & 2 & 2 & 82.23 & 0.0036 & 277.2613 & 43.71 & 34.05 & 81.81 & 64.06 \\
\hline 6 & 91 & 28 & 378 & 1 & 2 & 3 & 66.22 & 0.0039 & 254.3159 & 29.75 & 42.28 & 82.06 & 94.12 \\
\hline 7 & 101 & 28 & 2.149 & 1 & 3 & 1 & 83.95 & $2.382 \times 10^{-4}$ & $4.199 \times 10^{3}$ & 41.54 & 33.35 & 74.24 & 7.40 \\
\hline 8 & 102 & 28 & 2.149 & 1 & 3 & 2 & 70.21 & $2.551 \times 10^{-4}$ & $3.921 \times 10^{3}$ & 31.94 & 39.88 & 74.42 & 87.66 \\
\hline 9 & 103 & 28 & 2.149 & 1 & 3 & 3 & 62.82 & $2.507 \times 10^{-4}$ & $3.990 \times 10^{3}$ & 32.22 & 44.57 & 74.37 & 86.91 \\
\hline 10 & 141 & 28 & 364 & 2 & 1 & 1 & 44.78 & 2.8628 & 0.3493 & 79.80 & 62.50 & 03.82 & 35.00 \\
\hline 11 & 142 & 28 & 3.364 & 2 & 1 & 2 & 42.91 & 2.6400 & 0.3788 & 79.71 & 65.24 & 103.52 & 35.13 \\
\hline 12 & 143 & 28 & 364 & 2 & 1 & 3 & 41.44 & 2.4083 & 0.4152 & 79.58 & 67.55 & 103.18 & 35.19 \\
\hline 13 & 153 & 28 & 2.828 & 2 & 2 & 1 & 85.37 & 1541 & 6.4872 & 51.00 & 32.84 & 93.56 & 54.90 \\
\hline 14 & 154 & 28 & 2.828 & 2 & 2 & 2 & 71.17 & 0.1713 & 5.8368 & 43.79 & 39.37 & 93.91 & 63.94 \\
\hline 15 & 155 & 28 & 2.828 & 2 & 2 & 3 & 62.73 & 1787 & 5.5960 & 43.59 & 44.66 & 94.05 & 64.23 \\
\hline 16 & 165 & 28 & 2.556 & 2 & 3 & 1 & 186.4 & 0.0099 & 101.3382 & 136.1 & 15.01 & 84.79 & 20.57 \\
\hline 17 & 166 & 28 & 2.556 & 2 & 3 & 2 & 115.9 & 0.0152 & 65.7891 & 75.03 & 24.17 & 86.11 & 37.32 \\
\hline 18 & 167 & 28 & 2.556 & 2 & 3 & 3 & 89.27 & 0.0187 & 53.6128 & 51.99 & 31.36 & 86.74 & 53.86 \\
\hline 19 & 205 & 28 & 3.722 & 3 & 1 & 1 & 38.44 & 7.8319 & 0.1277 & 114.7 & 72.87 & 107.64 & 24.42 \\
\hline 20 & 206 & 28 & 3.722 & 3 & 1 & 2 & 37.81 & 7.2505 & 0.1379 & 114.5 & 74.06 & 107.3 & 24.45 \\
\hline 21 & 207 & 28 & 3.722 & 3 & 1 & 3 & 37.27 & 6.6946 & 0.1494 & 114.5 & 75.14 & 107.0 & 24.47 \\
\hline 22 & 217 & 28 & 3.130 & 3 & 2 & 1 & 55.58 & 1.0821 & 0.9242 & 62.62 & 50.39 & 100.3 & 44.71 \\
\hline 23 & 218 & 28 & 3.130 & 3 & 2 & 2 & 52.56 & 1.0673 & 0.9370 & 62.26 & 53.29 & 100.2 & 44.97 \\
\hline 24 & 219 & 28 & 3.130 & 3 & 2 & 3 & 50.16 & 1.0409 & 0.9607 & 62.02 & 55.84 & 100.2 & 45.15 \\
\hline 25 & 229 & 28 & 2.828 & 3 & 3 & 1 & 92.50 & 0.1456 & 6.8671 & 57.25 & 30.31 & 93.37 & 48.91 \\
\hline 26 & 230 & 28 & 2.828 & 3 & 3 & 2 & 79.68 & 0.1612 & 6.2044 & 46.00 & 35.18 & 93.71 & 60.87 \\
\hline 27 & 231 & 28 & 2.828 & 3 & 3 & 3 & 71.17 & 0.1713 & 5.8368 & 43.79 & 39.37 & 93.91 & 63.94 \\
\hline
\end{tabular}


Table 7 Optimality properties for OCCD with Full Factorial Replicates in k = 4 variables

\begin{tabular}{|c|c|c|c|c|c|c|c|c|c|c|c|c|c|}
\hline \multirow[b]{2}{*}{$\begin{array}{c}\text { Desig } \\
\text { n } \\
\text { type: } \\
\text { OCC } \\
\text { D }\end{array}$} & \multirow[b]{2}{*}{$\mathbf{N}$} & \multirow[b]{2}{*}{$\mathbf{P}$} & \multirow[b]{2}{*}{$\alpha$} & \multirow[b]{2}{*}{$r_{f}$} & \multirow[b]{2}{*}{$r_{\alpha}$} & \multirow[b]{2}{*}{$n_{c}$} & \multirow[b]{2}{*}{ Aopt } & \multicolumn{2}{|c|}{ Dopt } & \multirow[b]{2}{*}{ Gopt } & \multirow[b]{2}{*}{ Aeff } & \multirow[b]{2}{*}{ Deff } & \multirow[b]{2}{*}{ Geff } \\
\hline & & & & & & & & $\max \left|\left(\frac{X^{\prime} X}{N}\right)\right|$ & $\min \mid\left(\frac{X^{\prime} X}{N}\right)$ & & & & \\
\hline 1 & 25 & 15 & 1.414 & 1 & 1 & 1 & 35.88 & $2.951 \times 10^{-4}$ & $3.388 \times 10^{3}$ & 15.88 & 41.81 & 58.17 & 94.49 \\
\hline 2 & 26 & 15 & 1.483 & 1 & 1 & 2 & 33.23 & $3.937 \times 10^{-4}$ & $2.540 \times 10^{3}$ & 16.35 & 45.13 & 59.28 & 91.75 \\
\hline 3 & 27 & 15 & 1.547 & 1 & 1 & 3 & 31.35 & $4.924 \times 10^{-4}$ & $2.031 \times 10^{3}$ & 16.82 & 47.85 & 60.18 & 89.17 \\
\hline 4 & 33 & 15 & 1.321 & 1 & 2 & 1 & 35.22 & $5.666 \times 10^{-5}$ & $1.765 \times 10^{4}$ & 20.12 & 42.59 & 52.10 & 74.55 \\
\hline 5 & 34 & 15 & 1.353 & 1 & 2 & 2 & 34.50 & $5.812 \times 10^{-5}$ & $1.720 \times 10^{4}$ & 20.58 & 43.48 & 52.20 & 72.88 \\
\hline 6 & 35 & 15 & 1.384 & 1 & 2 & 3 & 33.94 & $5.899 \times 10^{-5}$ & $1.695 \times 10^{4}$ & 21.04 & 44.21 & 52.25 & 71.29 \\
\hline 7 & 41 & 15 & 1.266 & 1 & 3 & 1 & 37.58 & $1.074 \times 10^{-5}$ & $9.315 \times 10^{4}$ & 24.28 & 39.91 & 46.63 & 61.78 \\
\hline 8 & 42 & 15 & 1.286 & 1 & 3 & 2 & 37.37 & $1.034 \times 10^{-5}$ & $9.673 \times 10^{4}$ & 24.73 & 40.14 & 46.52 & 60.65 \\
\hline 9 & 43 & 15 & 1.306 & 1 & 3 & 3 & 37.21 & $9.973 \times 10^{-6}$ & $1.003 \times 10^{5}$ & 25.18 & 40.31 & 46.40 & 59.57 \\
\hline 10 & 41 & 15 & 1.453 & 2 & 1 & 1 & 45.99 & $3.075 \times 10^{-4}$ & $3.252 \times 10^{3}$ & 21.09 & 32.62 & 58.32 & 71.11 \\
\hline 11 & 42 & 15 & 1.527 & 2 & 1 & 2 & 40.71 & $5.094 \times 10^{-4}$ & $1.963 \times 10^{3}$ & 20.72 & 36.84 & 60.31 & 72.38 \\
\hline 12 & 43 & 15 & 1.596 & 2 & 1 & 3 & 36.82 & $7.803 \times 10^{-4}$ & $1.282 \times 10^{3}$ & 20.75 & 40.74 & 62.06 & 72.28 \\
\hline 13 & 49 & 15 & 1.378 & 2 & 2 & 1 & 37.59 & $2.486 \times 10^{-4}$ & $4.023 \times 10^{3}$ & 15.64 & 39.91 & 57.51 & 95.93 \\
\hline 14 & 50 & 15 & 1.414 & 2 & 2 & 2 & 35.88 & $2.947 \times 10^{-4}$ & $3.394 \times 10^{3}$ & 15.88 & 41.81 & 58.17 & 94.49 \\
\hline 15 & 51 & 15 & 1.449 & 2 & 2 & 3 & 34.44 & $3.433 \times 10^{-4}$ & $2.913 \times 10^{3}$ & 16.11 & 43.55 & 5876 & 93.10 \\
\hline 16 & 57 & 15 & 1.336 & 2 & 3 & 1 & 35.65 & $1.248 \times 10^{-4}$ & $8.015 \times 10^{3}$ & 17.78 & 42.07 & 54.92 & 84.38 \\
\hline 17 & 58 & 15 & 1.359 & 2 & 3 & 2 & 34.85 & $1.331 \times 10^{-4}$ & $7.513 \times 10^{3}$ & 18.01 & 43.04 & 55.16 & 83.29 \\
\hline 18 & 59 & 15 & 1.382 & 2 & 3 & 3 & 34.15 & $1.416 \times 10^{-4}$ & $7.062 \times 10^{3}$ & 18.24 & 43.93 & 55.37 & 82.22 \\
\hline 19 & 57 & 15 & 1.467 & 3 & 1 & 1 & 57.76 & $1.767 \times 10^{-4}$ & $5.659 \times 10^{3}$ & 28.26 & 25.97 & 56.22 & 53.08 \\
\hline 20 & 58 & 15 & 1.543 & 3 & 1 & 2 & 60.18 & $2.222 \times 10^{-4}$ & $4.500 \times 10^{3}$ & 27.39 & 29.99 & 58.51 & 54.77 \\
\hline 21 & 59 & 15 & 1.615 & 3 & 1 & 3 & 64.39 & $2.647 \times 10^{-4}$ & $3777 \times 10^{3}$ & 27.10 & 33.89 & 60.58 & 55.35 \\
\hline 22 & 65 & 15 & 1.402 & 3 & 2 & 1 & 43.07 & $2.820 \times 10^{-4}$ & $3.546 \times 10^{3}$ & 17.76 & 34.83 & 57.98 & 84.47 \\
\hline 23 & 66 & 15 & 1.439 & 3 & 2 & 2 & 40.51 & $3.572 \times 10^{-4}$ & $2.800 \times 10^{3}$ & 17.53 & 37.03 & 58.92 & 85.57 \\
\hline 24 & 67 & 15 & 1.476 & 3 & 2 & 3 & 38.35 & $4.468 \times 10^{-4}$ & $2.238 \times 10^{3}$ & 17.42 & 39.11 & 59.78 & 86.09 \\
\hline 25 & 73 & 15 & 1.366 & 3 & 3 & 1 & 38.23 & $2.343 \times 10^{-4}$ & $4.268 \times 10^{3}$ & 15.56 & 39.24 & 57.28 & 96.41 \\
\hline 26 & 74 & 15 & 1.390 & 3 & 3 & 2 & 36.98 & $2.634 \times 10^{-4}$ & $3.797 \times 10^{3}$ & 15.72 & 40.56 & 57.74 & 95.44 \\
\hline 27 & 75 & 15 & 1.414 & 3 & 3 & 3 & 40.80 & $1.304 \times 10^{-4}$ & $7.671 \times 10^{3}$ & 15.88 & 41.81 & 58.17 & 94.49 \\
\hline
\end{tabular}


Table 8 Optimality properties for OCCD with Full Factorial Replicates in $k=5$ variables

\begin{tabular}{|c|c|c|c|c|c|c|c|c|c|c|c|c|c|}
\hline \multirow[b]{2}{*}{$\begin{array}{l}\text { Design } \\
\text { type: } \\
\text { OCCD }\end{array}$} & \multirow[b]{2}{*}{$\mathbf{N}$} & \multirow[b]{2}{*}{$\mathbf{P}$} & \multirow[b]{2}{*}{$\boldsymbol{\alpha}$} & \multirow[b]{2}{*}{$r_{f}$} & \multirow[b]{2}{*}{$r_{\alpha}$} & \multirow[b]{2}{*}{$n_{c}$} & \multirow[b]{2}{*}{ Aopt } & \multicolumn{2}{|c|}{ Dopt } & \multirow[b]{2}{*}{ Gopt } & \multirow[b]{2}{*}{ Aeff } & \multirow[b]{2}{*}{ Deff } & \multirow[b]{2}{*}{ Geff } \\
\hline & & & & & & & & $\max \left|\left(\frac{X^{\prime} X}{N}\right)\right|$ & $\min \mid\left(\frac{X^{\prime} X}{N}\right)$ & & & & \\
\hline 1 & 43 & 21 & 596 & 1 & 1 & 1 & 49.13 & $6.230 \times 10^{-5}$ & $1.605 \times 10^{4}$ & 23.22 & 42.74 & 63.06 & 90.44 \\
\hline 2 & 44 & 1 & 662 & 1 & 1 & 2 & 45.52 & $9.365 \times 10^{-5}$ & $1.068 \times 10^{4}$ & .14 & 46.13 & & .75 \\
\hline 3 & 45 & 1 & 724 & 1 & 1 & 3 & 4.77 & $317 \times 10^{-4}$ & $7.595 \times 10^{3}$ & 23.31 & 49.11 & 65.36 & .09 \\
\hline 4 & 53 & & 515 & 1 & 2 & 1 & & $813 \mathrm{X}$ & $5.517 \times 10^{4}$ & 24.62 & 47.56 & 59.46 & .29 \\
\hline 5 & 54 & & 547 & 1 & 2 & 2 & & $1.983 \times 10^{-5}$ & $5.044 \times 10^{4}$ & 24.99 & 48.66 & 59.70 & \\
\hline 6 & 55 & 1 & 7 & 1 & 2 & 3 & & $2.115 \times 10^{-5}$ & $4.728 \times 10^{4}$ & 25.37 & 49.64 & 91 & .78 \\
\hline 7 & 63 & & & 1 & 3 & 1 & & & $2.886 \times 10^{5}$ & & 46.84 & 6 & .32 \\
\hline 8 & \begin{tabular}{|l}
64 \\
\end{tabular} & & 486 & 1 & 3 & 2 & 4.47 & $449 \times 10^{-6}$ & $2.900 \times 10^{5}$ & 29.01 & 47.23 & 54.95 & 2.39 \\
\hline 9 & & & 506 & & 3 & 3 & & $32 \mathrm{X}$ & $2.914 \times 10^{5}$ & 29.38 & 47.56 & & \\
\hline 10 & 75 & 1 & 625 & 2 & 1 & 1 & 65 & $2.112 \mathrm{X}$ & $4.734 \times 10^{4}$ & 07 & 30.90 & & \\
\hline 11 & 76 & & 695 & 2 & 1 & 2 & 8 & $34 X$ & $1.775 \times 10^{4}$ & 37.25 & 34.55 & 74 & 6.38 \\
\hline 12 & 77 & & 761 & & 1 & 3 & & $28 \mathrm{X}$ & $1.039 \times 10^{4}$ & 36 & 38.04 & & .90 \\
\hline 13 & 85 & 1 & 562 & 2 & 2 & 1 & 35 & $0^{-5}$ & $2.014 \times 10^{4}$ & 23.39 & 40.90 & 38 & 9.79 \\
\hline 14 & o & & 596 & & 2 & 2 & & 60200 & $1.605 \times 10^{4}$ & & 42.74 & & 0.44 \\
\hline 15 & 87 & 21 & 9 & 2 & 2 & 3 & & & $1.304 \times 10^{4}$ & & 44.49 & 70 & .74 \\
\hline 16 & 95 & & 526 & & 3 & 1 & & $82 X$ & $2.957 \times 10^{4}$ & 22.40 & 45.30 & 25 & 3.73 \\
\hline 17 & 96 & 1 & 548 & 2 & 3 & 2 & & $3.751 \times 10^{-5}$ & $2.666 \times 10^{4}$ & 22.59 & 46.33 & & .95 \\
\hline 18 & 97 & 21 & 570 & 2 & 3 & 3 & 44.39 & $4.146 \mathrm{X}$ & $2.412 \times 10^{4}$ & 22.78 & 47.31 & 61.85 & 2.18 \\
\hline 19 & & & 636 & & 1 & & & $1.108 \times 10^{-5}$ & $9.028 \times 10^{4}$ & 49.84 & 20.51 & & 2.13 \\
\hline 20 & & & 706 & 3 & 1 & 2 & & $2.182 \mathrm{X}$ & $4.584 \times 10^{4}$ & 51.43 & 27.02 & 59.99 & 0.83 \\
\hline 21 & & & 774 & & 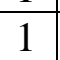 & 2 & 69.44 & $4.051 \times 10^{-5}$ & $2.469 \times 10^{4}$ & 53.34 & 21.93 & 59.97 & 9.37 \\
\hline 22 & & 21 & .580 & 3 & 2 & 1 & 61.47 & $3.698 \times 10^{-5}$ & $2.704 \times 10^{4}$ & 31.04 & 34.16 & 61.50 & 7.65 \\
\hline 23 & 118 & 21 & .615 & 5 & 2 & 2 & 58.16 & $4.946 \times 10^{-5}$ & $2.022 \times 10^{4}$ & 30.63 & 36.11 & 37 & 8.57 \\
\hline 24 & & & 650 & 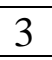 & - & 3 & 55.27 & $6.539 \times 10^{-5}$ & $1.529 \times 10^{4}$ & 30.35 & 38.00 & 63.19 & 9.20 \\
\hline 25 & 12 & 2 & 1.550 & 3 & 3 & 1 & 52.17 & $4.565 \times 10^{-5}$ & $2.191 \times 10^{4}$ & 23.47 & 40.26 & 62.13 & 9.48 \\
\hline 26 & 128 & 21 & 1.573 & $J$ & 3 & 2 & 50.58 & $5.345 \times 10^{-5}$ & $1.871 \times 10^{4}$ & 23.32 & 41.53 & 62.61 & 0.05 \\
\hline 27 & 129 & 21 & 1.596 & 3 & 3 & 3 & 49.13 & $6.230 \times 10^{-5}$ & $1.605 \times 10^{4}$ & 23.22 & 42.74 & 63.06 & 90.44 \\
\hline
\end{tabular}


Table 9 Optimality properties for SCCD with Full Factorial Replicates in $k=6$ variables

\begin{tabular}{|c|c|c|c|c|c|c|c|c|c|c|c|c|c|}
\hline \multirow{2}{*}{\begin{tabular}{|c|} 
Design \\
type: \\
OCC \\
D \\
\end{tabular}} & \multirow[b]{2}{*}{$\mathbf{N}$} & \multirow[b]{2}{*}{$\mathbf{P}$} & \multirow[b]{2}{*}{$\boldsymbol{\alpha}$} & \multirow[b]{2}{*}{$r_{f}$} & \multirow[b]{2}{*}{$r_{\alpha}$} & \multirow[b]{2}{*}{$n_{c}$} & \multirow[b]{2}{*}{ Aopt } & \multicolumn{2}{|c|}{ Dopt } & \multirow[b]{2}{*}{ Gopt } & \multirow[b]{2}{*}{ Aeff } & \multirow[b]{2}{*}{ Deff } & \multirow[b]{2}{*}{ Geff } \\
\hline & & & & & & & & $\max \left|\left(\frac{X^{\prime} X}{N}\right)\right|$ & $\min \mid\left(\frac{\Lambda \Lambda}{N}\right.$ & & & & \\
\hline 1 & 77 & & 761 & 1 & 1 & 1 & 69.65 & $8.664 \times 10^{-6}$ & $1.154 \times 10^{5}$ & 40.24 & 40.20 & 65.95 & 69.59 \\
\hline 2 & 78 & & .824 & 1 & 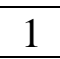 & 2 & 4.39 & $1.485 \times 10^{-5}$ & $6.733 \times 10^{4}$ & 9.78 & 43.49 & 67.23 & 0.39 \\
\hline 3 & 79 & & 1.885 & 1 & 1 & & 0.17 & $2.408 \times 10^{-5}$ & $.152 \times 10^{4}$ & 9.64 & 46.54 & 40 & 70.63 \\
\hline 4 & 89 & & 1.694 & 1 & 2 & & 6.84 & $6.784 \times 10^{-6}$ & $474 \times 10^{5}$ & 9.31 & 49.26 & 65.36 & 95.53 \\
\hline 5 & 90 & 8 & 1.724 & 1 & 2 & & 5.33 & $7.929 \times 10^{-6}$ & $261 \times 10^{5}$ & 9.59 & 50.60 & & 4.65 \\
\hline 6 & 91 & & .755 & 1 & 2 & & .01 & $9.308 \times 10^{-6}$ & $1.074 \times 10^{5}$ & 9.86 & & & .78 \\
\hline 7 & 101 & & 1.653 & 1 & 3 & & 0 & $7 \times 10^{-6}$ & $839 \times 10^{5}$ & & & & דיד. \\
\hline 8 & 2 & & 1.673 & 1 & 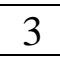 & & 7 & $\mathrm{X} 10^{-6}$ & $.594 \times 10^{5}$ & 04 & 52.18 & 62.78 & 84.74 \\
\hline 9 & 3 & & 1.693 & 1 & 3 & & 3.09 & $X 10^{-6}$ & $.370 \times 10^{5}$ & 32 & 74 & & UJ \\
\hline 10 & 41 & & 1.781 & 2 & 1 & & 104.1 & X $10^{-6}$ & $.755 \times 10^{5}$ & 70.65 & 26.91 & 61.91 & .63 \\
\hline 11 & 2 & & 846 & 2 & & & & $\times 10^{-6}$ & $379 \times 10^{5}$ & & & & 40.53 \\
\hline 12 & 3 & & & 2 & 1 & & & X $10^{-6}$ & $1.770 \times 10^{5}$ & & & 94 & \\
\hline 13 & 53 & & 1.728 & 2 & 2 & & 2.77 & X $10^{-6}$ & $.547 \times 10^{5}$ & 40.62 & 38.48 & 65.26 & 94 \\
\hline 14 & & & .761 & 2 & 2 & & & X $10^{-6}$ & $1.150 \times 10^{5}$ & 40.24 & 40.20 & 65.95 & 69.59 \\
\hline 15 & 155 & & 1.793 & 2 & 2 & 3 & 5.90 & $7 \times 10^{-5}$ & $.717 \times 10^{4}$ & 39.96 & 41.87 & 66.61 & 70.07 \\
\hline 16 & & & 1.699 & 2 & & & & $2 \times 10^{-6}$ & $247 \times 10^{5}$ & & 44.96 & 65.78 & 92.25 \\
\hline 17 & 6 & & 721 & 2 & 3 & & & X $10^{-6}$ & $.060 \times 10^{5}$ & & 04 & 14 & 03 \\
\hline 18 & 167 & & 1.742 & 2 & 3 & & 9.47 & $3 \times 10^{-5}$ & $9.151 \times 10^{4}$ & 30.14 & 47.09 & 66.50 & 92.90 \\
\hline 19 & 205 & & .788 & 3 & 1 & & & $2 \times 10^{-7}$ & $3.309 \times 10^{6}$ & 101.1 & 20.03 & 58.50 & 27.65 \\
\hline 20 & 206 & 8 & 854 & 3 & 1 & 0 & 24.3 & & $X 10^{6}$ & 98.44 & 22.53 & .10 & 28.44 \\
\hline 21 & 207 & & 918 & 3 & & & 111.9 & $1 \times 10^{-6}$ & $.746 \times 10^{5}$ & 96.68 & 25.04 & 61.62 & 28.96 \\
\hline 22 & 217 & & 1.741 & 3 & 2 & & & $0 \times 10^{-6}$ & $3.845 \times 10^{5}$ & 56.16 & 30.71 & 63.16 & 49.86 \\
\hline 23 & 218 & & 1.774 & 3 & 2 & & 86.53 & $1 \times 10^{-6}$ & $2.731 \times 10^{5}$ & 55.43 & 32.36 & 63.95 & 50.52 \\
\hline 2 & 219 & & 1.807 & 3 & 2 & & & $5.101 \times 10^{-6}$ & $1.961 \times 10^{5}$ & 54.86 & 33.99 & 64.71 & 51.04 \\
\hline 25 & 229 & & 1.717 & 3 & 3 & & 73.90 & $5.842 \times 10^{-6}$ & $1.712 \times 10^{5}$ & 40.77 & 37.89 & 65.02 & 68.68 \\
\hline 26 & 230 & & .739 & 3 & 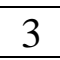 & & 71.69 & $7.144 \times 10^{-6}$ & $1.400 \times 10^{5}$ & 40.48 & 39.06 & 65.49 & 69.18 \\
\hline 27 & 231 & 20 & 1.761 & 3 & 3 & 3 & 69.65 & $8.696 \times 10^{-6}$ & $1.150 \times 10^{5}$ & 40.24 & 40.20 & 65.95 & 69.59 \\
\hline
\end{tabular}


Table 10 Optimality properties for FCCD with Full Factorial Replicates in $k=4$ variables

\begin{tabular}{|c|c|c|c|c|c|c|c|c|c|c|c|c|c|}
\hline \multirow[b]{2}{*}{$\begin{array}{l}\text { Design } \\
\text { type: } \\
\text { FCCD }\end{array}$} & \multirow[b]{2}{*}{$\mathbf{N}$} & \multirow[b]{2}{*}{$\mathbf{P}$} & \multirow[b]{2}{*}{ c } & \multirow[b]{2}{*}{$r_{f}$} & \multirow[b]{2}{*}{$r_{\alpha}$} & \multirow[b]{2}{*}{$n_{c}$} & \multirow[b]{2}{*}{ Aopt } & \multicolumn{2}{|c|}{ Dopt } & \multirow[b]{2}{*}{ Gopt } & \multirow[b]{2}{*}{ Aeff } & \multirow[b]{2}{*}{$D e f f$} & \multirow[b]{2}{*}{ Geff } \\
\hline & & & & & & & & $\max \left|\left(\frac{X^{\prime} X}{N}\right)\right|$ & $\min \mid\left(\frac{X^{\prime} X}{N}\right)$ & & & & \\
\hline 1 & 25 & 15 & 1 & 3 & 1 & 1 & 58.86 & $5.356 \times 10^{-6}$ & $1.867 \times 10^{5}$ & 16.48 & 25.49 & 44.52 & 91.00 \\
\hline 2 & 26 & & & & 1 & 2 & & $3.528 \times 10^{-6}$ & $2.834 \times 10^{5}$ & 17.14 & 24.91 & 43.30 & 87.53 \\
\hline 3 & 27 & 5 & & & 1 & 3 & 1.79 & $2.318 \times 10^{-6}$ & $4.314 \times 10^{5}$ & 17.79 & 24.28 & 42.11 & 84.31 \\
\hline 4 & 3 & & & & 2 & 1 & 48.90 & $1.846 \times 10^{-6}$ & $5.418 \times 10^{5}$ & 21.03 & 30.68 & 41.47 & 71.35 \\
\hline 5 & 34 & & & & 2 & 2 & 49.92 & $1.304 \times 10^{-6}$ & $7.668 \times 10^{5}$ & 21.65 & 30.05 & 40.52 & 69.28 \\
\hline 6 & 25 & & & & 0 & & 51.00 & $9.249 \times 10^{-7}$ & $1.081 \times 10^{6}$ & 22.28 & 29.41 & 39.60 & 67.33 \\
\hline 7 & & & & & 3 & 1 & 48.20 & $5.099 \times 10^{-7}$ & $1.961 \times 10^{6}$ & 25.38 & 31.12 & & 59.11 \\
\hline 8 & 40 & & & & 3 & 2 & 49.07 & $3.818 \times 10^{-7}$ & $2.619 \times 10^{6}$ & 25.98 & 30.57 & 37.34 & 57.74 \\
\hline 9 & & & & & $J$ & 3 & 49.97 & $2.870 \times 10^{-7}$ & $3.485 \times 10^{6}$ & 26.59 & 30.02 & 36.63 & 56.42 \\
\hline 10 & & & & & 1 & 1 & 97 & $1 \times 10^{-6}$ & $3.485 \times 10^{6}$ & 20.78 & 17.87 & 44.44 & 72.20 \\
\hline 11 & 41 & & & & 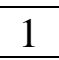 & 2 & 46 & $\times 10^{-6}$ & $2.326 \times 10^{5}$ & 20.63 & 17.76 & 43.88 & 72.72 \\
\hline 12 & & & & & 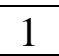 & 3 & & $\mathrm{X} \mathrm{10}=6$ & $2.865 \times 10^{5}$ & 20.63 & 17.48 & & 72.72 \\
\hline 13 & 49 & & & & 2 & 1 & 48.29 & $6.575 \times 10^{-6}$ & $1.521 \times 10^{5}$ & 16.16 & 25.73 & 45.14 & 92.83 \\
\hline 14 & 50 & & & & 2 & & 58.86 & $5.356 \times 10^{-6}$ & $1.867 \times 10^{5}$ & & 25.49 & 44.52 & 91.00 \\
\hline 15 & 51 & & & & 2 & 3 & & & $2.299 \times 10^{5}$ & & 25.21 & 43.91 & 89.23 \\
\hline 16 & 57 & & & & & 1 & 1.03 & $4.134 \times 10^{-6}$ & $2.419 \times 10^{5}$ & 18.46 & 29.39 & 43.76 & 81.24 \\
\hline 17 & & & 1 & & 3 & 2 & & $3.414 \times 10^{-6}$ & $2.930 \times 10^{5}$ & 18.78 & 29.09 & 43.21 & 79.86 \\
\hline$\overline{18}$ & 59 & 15 & 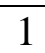 & & 3 & 3 & 52.11 & $2.819 \times 10^{-6}$ & $3.547 \times 10^{5}$ & 19.10 & 28.78 & 42.66 & 78.53 \\
\hline 19 & & & & & & & 110.7 & $2.970 \times 10^{-6}$ & $3.367 \times 10^{5}$ & 28.35 & 13.55 & 42.81 & 52.92 \\
\hline 20 & 58 & & 1 & & 1 & 2 & 110.6 & $2.708 \times 10^{-6}$ & $3.693 \times 10^{5}$ & 27.94 & 13.57 & 42.54 & 53.69 \\
\hline 21 & 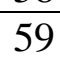 & & 1 & & 1 & & 110.9 & $2.420 \times 10^{-6}$ & $4.132 \times 10^{5}$ & 27.74 & 13.52 & 42.23 & 54.07 \\
\hline 22 & 05 & & 1 & & 2 & 1 & 70.69 & $7.049 \times 10^{-6}$ & $1.419 \times 10^{5}$ & 17.10 & 21.22 & 45.35 & 87.75 \\
\hline 23 & 66 & & 1 & & 2 & 2 & 70.98 & $6.178 \times 10^{-6}$ & $1.619 \times 10^{5}$ & & 21.13 & 44.95 & 88.12 \\
\hline 24 & 67 & & 1 & & 2 & & 71.39 & $5.386 \times 10^{-6}$ & $1.857 \times 10^{5}$ & 17.00 & 21.01 & 44.54 & 88.25 \\
\hline 25 & 73 & 1 & 1 & & 3 & 1 & 58.12 & $7.035 \times 10^{-6}$ & $1.422 \times 10^{5}$ & 16.05 & 25.81 & 45.34 & 93.46 \\
\hline 26 & 74 & 1 & 1 & 3 & 3 & 2 & 58.47 & $6.143 \times 10^{-6}$ & $1.628 \times 10^{5}$ & 16.27 & 25.66 & 44.93 & 92.21 \\
\hline 27 & 75 & 15 & 1 & $J$ & 3 & 3 & 58.86 & $5.356 \times 10^{-6}$ & $1.867 \times 10^{5}$ & 16.48 & 25.49 & 44.52 & 91.00 \\
\hline
\end{tabular}


Table 11 Optimality properties for FCCD with Full Factorial Replicates in $\mathbf{k}=\mathbf{5}$ variables

\begin{tabular}{|c|c|c|c|c|c|c|c|c|c|c|c|c|c|}
\hline \multirow[b]{2}{*}{$\begin{array}{c}\text { Design } \\
\text { type: } \\
\text { FCCD }\end{array}$} & \multirow[b]{2}{*}{$\mathbf{N}$} & \multirow[b]{2}{*}{$\mathbf{P}$} & \multirow[b]{2}{*}{$\alpha$} & \multirow[b]{2}{*}{$r_{f}$} & \multirow[b]{2}{*}{$r_{\alpha}$} & \multirow[b]{2}{*}{$n_{c}$} & \multirow[b]{2}{*}{ Aopt } & \multicolumn{2}{|c|}{ Dopt } & \multirow[b]{2}{*}{ Gopt } & \multirow[b]{2}{*}{ Aeff } & \multirow[b]{2}{*}{$D e f f$} & \multirow[b]{2}{*}{ Geff } \\
\hline & & & & & & & & $\max \left|\left(\frac{X^{\prime} X}{N}\right)\right|$ & $\min \mid\left(\frac{X^{\prime} X}{N}\right)$ & & & & \\
\hline 1 & 43 & 21 & 1 & 1 & 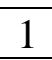 & 1 & 113.2 & $4.834 \times 10^{-8}$ & $2.069 \times 10^{7}$ & 22.19 & 18.55 & 44.84 & 94.66 \\
\hline 2 & 44 & 21 & 1 & 1 & 1 & 2 & 115.0 & $3.390 \times 10^{-8}$ & $2.950 \times 10^{7}$ & 22.25 & 18.27 & 44.09 & 94.38 \\
\hline 3 & 45 & 21 & & & & 3 & 116.9 & $2.369 \times 10^{-8}$ & $4.222 \times 10^{7}$ & 22.39 & 17.97 & 43.34 & 93.78 \\
\hline 4 & 53 & 21 & & & & 1 & & $2.379 \times 10^{-8}$ & $4.205 \times 10^{7}$ & 25.58 & 25.57 & 43.35 & 82.11 \\
\hline 5 & 54 & 21 & 1 & & & 2 & 83.33 & $1.725 \times 10^{-8}$ & $5.797 \times 10^{7}$ & 26.06 & 25.20 & 42.69 & 80.59 \\
\hline 6 & 55 & 21 & & & & 3 & 84.57 & $1.255 \times 10^{-8}$ & $7.971 \times 10^{7}$ & 26.54 & 24.83 & 40.05 & 79.14 \\
\hline 7 & 63 & 21 & & & & 1 & 74.43 & $6.126 \times 10^{-9}$ & $1.633 \times 10^{8}$ & 29.94 & 28.21 & 40.64 & 70.14 \\
\hline 8 & 64 & 21 & 1 & & & 2 & 75.41 & $4.626 \times 10^{-9}$ & $2.162 \times 10^{8}$ & 30.41 & 27.85 & 40.10 & 69.05 \\
\hline 9 & 65 & 21 & & & & 3 & 76.40 & $X 10^{-9}$ & $2.854 \times 10^{8}$ & 30.89 & 27.49 & 39.57 & 67.99 \\
\hline 10 & 75 & 21 & 1 & & & 1 & 180.0 & $2.302 \times 10^{-8}$ & $4.344 \times 10^{7}$ & 37.62 & 11.67 & 43.28 & 55.82 \\
\hline 11 & 76 & 21 & & & & 2 & 180.9 & $1.980 \times 10^{-8}$ & $5.051 \times 10^{7}$ & 37.35 & 11.61 & 42.97 & 56.23 \\
\hline 12 & 77 & 21 & 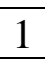 & & & 3 & 182.0 & $1.685 \times 10^{-8}$ & $5.936 \times 10^{7}$ & 37.21 & 11.54 & 42.64 & 56.43 \\
\hline 13 & 85 & 21 & & & & 1 & 112.4 & $5.757 \times 10^{-8}$ & $1.737 \times 10^{7}$ & 22.19 & 18.68 & 45.21 & 94.63 \\
\hline 14 & 86 & 21 & & & & 2 & 113.2 & $4.834 \times 10^{-8}$ & $2.069 \times 10^{7}$ & 22.19 & 18.55 & 44.84 & 94.66 \\
\hline 15 & 87 & 21 & 1 & & & 3 & 114.1 & $4.051 \times 10^{-8}$ & $2.468 \times 10^{7}$ & 22.21 & 18.41 & 44.46 & 94.57 \\
\hline 16 & 95 & 21 & 1 & & & 1 & 91.14 & $4.771 \times 10^{-8}$ & $2.096 \times 10^{-7}$ & 23.11 & 23.04 & 44.81 & 90.86 \\
\hline 17 & 96 & 21 & 1 & 2 & 3 & 2 & & $X 10^{-8}$ & $2.486 \times 10^{7}$ & 23.36 & 22.87 & 44.45 & 89.92 \\
\hline 18 & 97 & 21 & 1 & & & 3 & 92.51 & $3.391 \times 10^{-8}$ & $2.949 \times 10^{7}$ & 23.60 & 22.70 & 44.09 & 89.00 \\
\hline 19 & 07 & 21 & 1 & 3 & & 1 & & $8.253 \times 10^{=9}$ & $1.212 \times 10^{8}$ & 53.15 & 8.456 & 41.22 & 39.51 \\
\hline 20 & 08 & 21 & 1 & 3 & 1 & 2 & 248.6 & $7.709 \times 10^{-9}$ & $1.297 \times 10^{8}$ & 52.53 & 8.449 & 41.08 & 39.98 \\
\hline 21 & 109 & 21 & 1 & & & 3 & 249.2 & $7.111 \times 10^{-9}$ & $1.406 \times 10^{8}$ & 52.14 & 8.428 & 40.93 & 40.28 \\
\hline 22 & 117 & 21 & 1 & 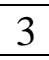 & & 1 & 145.7 & $4.173 \times 10^{-8}$ & $2.396 \times 10^{7}$ & 30.00 & 14.42 & 44.52 & 70.01 \\
\hline 23 & 118 & 21 & 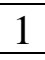 & 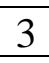 & & 2 & 146.2 & $3.745 \times 10^{-8}$ & $2.670 \times 10^{7}$ & 29.88 & 14.37 & 44.30 & 70.28 \\
\hline 24 & 19 & 21 & 1 & 3 & 2 & 3 & 146.8 & $3.350 \times 10^{-8}$ & $2.985 \times 10^{7}$ & 29.81 & 14.31 & 44.06 & 70.44 \\
\hline 25 & 127 & 21 & 1 & 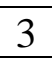 & 3 & 1 & 112.2 & $6.100 \times 10^{-8}$ & $1.640 \times 10^{7}$ & 22.20 & 18.73 & 45.34 & 94.59 \\
\hline 26 & 128 & 21 & 1 & 3 & 3 & 2 & 112.7 & $5.433 \times 10^{-8}$ & $1.841 \times 10^{7}$ & 22.19 & 18.64 & 45.09 & 94.65 \\
\hline 27 & 129 & 21 & 1 & 3 & 3 & 3 & 113.2 & $4.834 \times 10^{-8}$ & $2.069 \times 10^{7}$ & 22.19 & 18.55 & 44.84 & 94.66 \\
\hline
\end{tabular}


Table 12 Optimality properties for FCCD with Full Factorial Replicates in $k=6$ variables

\begin{tabular}{|c|c|c|c|c|c|c|c|c|c|c|c|c|c|}
\hline \multirow{2}{*}{$\begin{array}{l}\text { Design } \\
\text { type: } \\
\text { FCCD }\end{array}$} & \multirow[b]{2}{*}{$\mathbf{N}$} & \multirow[b]{2}{*}{$\mathbf{P}$} & \multirow[b]{2}{*}{$\alpha$} & \multirow[b]{2}{*}{$r_{f}$} & \multirow[b]{2}{*}{$r_{\alpha}$} & \multirow[b]{2}{*}{$n_{c}$} & \multirow[b]{2}{*}{ Aopt } & \multicolumn{2}{|c|}{ Dopt } & \multirow[b]{2}{*}{ Gopt } & \multirow[b]{2}{*}{ Aeff } & \multirow[b]{2}{*}{ Deff } & \multirow[b]{2}{*}{ Geff } \\
\hline & & & & & & & & $\max \left|\left(\frac{X^{\prime} X}{N}\right)\right|$ & $\min \mid\left(\frac{X^{\prime} X}{N}\right)$ & & & & \\
\hline 1 & 77 & 28 & 0000 & 2 & 1 & 1 & 27.5 & $1.770 \times 10^{-10}$ & $5.650 \times 10^{9}$ & 38.98 & 12.31 & 4.85 & 71.83 \\
\hline 2 & 78 & 8 & 000 & 1 & 1 & 2 & 29.5 & $1.366 \times 10^{-10}$ & $7.320 \times 10^{9}$ & 3 & 2.20 & .43 & .93 \\
\hline 3 & 79 & 28 & 0000 & 1 & 1 & 3 & 231.6 & $1.049 \times 10^{-10}$ & $9.531 \times 10^{9}$ & 38.96 & 12.09 & 44.02 & 1.88 \\
\hline 4 & 89 & 28 & 0000 & 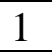 & 2 & 1 & 146.2 & $2.223 \times 10^{-10}$ & $4.499 \times 10^{9}$ & 30.10 & 19.15 & 45.21 & 3.02 \\
\hline 5 & 90 & 28 & 0000 & 1 & 2 & 2 & 147.5 & $1.719 \times 10^{-10}$ & $5.819 \times 10^{9}$ & 0.44 & 18.98 & .80 & 1.99 \\
\hline 6 & 91 & 28 & 0000 & 1 & 2 & 3 & 48.9 & $1.329 \times 10^{-10}$ & $7.522 \times 10^{9}$ & 30.78 & 18.81 & 4.39 & 90.98 \\
\hline 7 & 101 & 8 & 0000 & 1 & 3 & 1 & 121.5 & $8.563 \times 10^{-11}$ & $1.168 \times 10^{10}$ & 33.91 & 23.05 & 44.70 & 2.58 \\
\hline 8 & 102 & 8 & 0000 & 1 & 3 & 2 & & $6.752 \times 10^{-11}$ & $1.481 \times 10^{10}$ & 4.24 & 22.86 & .33 & 1.77 \\
\hline 9 & 3 & 8 & 0000 & 1 & 3 & 3 & & $\mathrm{X} 10^{-11}$ & $1.876 \times 10^{10}$ & 34.58 & 22.67 & .96 & .98 \\
\hline 10 & 141 & & 000 & 2 & 1 & 1 & & $2.981 \times 10^{-11}$ & $3.355 \times 10^{10}$ & 33 & 1.12 & .08 & 39.82 \\
\hline 11 & 142 & 8 & 0000 & 2 & 1 & 2 & 94.5 & $3 \times 10^{-11}$ & $3.692 \times 10^{10}$ & 69.80 & 7.10 & 41.94 & 40.11 \\
\hline 12 & 143 & 8 & 0000 & 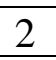 & 1 & 3 & 395.9 & $2.441 \times 10^{-11}$ & $4.097 \times 10^{10}$ & 9.45 & 7.07 & 41.78 & 40.32 \\
\hline 13 & 153 & 8 & 0000 & 2 & 2 & 1 & 226.6 & $\mathrm{X} 10^{-10}$ & $4.975 \times 10^{9}$ & .05 & 12.36 & 5.05 & 71.71 \\
\hline 14 & 154 & 8 & 0000 & - & 2 & 2 & 227.5 & $1.770 \times 10^{-10}$ & $5.650 \times 10^{9}$ & 98 & 12.31 & 85 & 71.83 \\
\hline 15 & 155 & & & - & 2 & 3 & 28.5 & $1.556 \times 10^{-10}$ & $6.426 \times 10^{9}$ & 38.94 & 12.26 & .64 & 71.91 \\
\hline 16 & 165 & 28 & 0000 & 2 & 3 & 1 & 172.0 & $2.968 \times 10^{-10}$ & $3.369 \times 10^{9}$ & 28.56 & 16.28 & 45.68 & 98.06 \\
\hline 17 & 166 & 28 & 0000 & 2 & 3 & 2 & 172.7 & $2.603 \times 10^{-10}$ & $3.842 \times 10^{9}$ & 28.56 & 16.21 & 45.47 & 98.03 \\
\hline 18 & 167 & 28 & .0000 & 2 & 3 & 3 & 173.5 & $\mathrm{X} 10^{-10}$ & $4.382 \times 10^{9}$ & 28.58 & 16.14 & 5.25 & 97.96 \\
\hline 19 & 205 & 28 & 0000 & 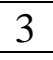 & 1 & 1 & 560.8 & $\mathrm{X} 10^{-12}$ & $1.645 \times 10^{11}$ & 101.73 & 4.99 & 39.76 & 27.53 \\
\hline 20 & 206 & 20 & 0000 & 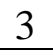 & 1 & 2 & 561.0 & $5.875 \times 10^{-12}$ & $1.702 \times 10^{10}$ & 100.74 & 4.99 & 3971 & 27.79 \\
\hline 21 & 207 & 28 & 1.0000 & 3 & 1 & 3 & 561.6 & $5.628 \times 10^{-12}$ & $1.778 \times 10^{11}$ & 100.00 & 4.99 & 39.65 & 28.00 \\
\hline 22 & 217 & 28 & 1.0000 & 3 & 2 & 1 & 309.5 & $7.965 \times 10^{-11}$ & $1.256 \times 10^{10}$ & 54.85 & 9.05 & 43.58 & 51.05 \\
\hline 23 & 218 & 28 & 1.0000 & 3 & 2 & 2 & & $7.400 \times 10^{-11}$ & $1.351 \times 10^{10}$ & 54.64 & 9.03 & 43.47 & 51.25 \\
\hline 24 & 219 & 28 & 1.0000 & 3 & 2 & 3 & 310.9 & $6.860 \times 10^{-11}$ & $1.458 \times 10^{10}$ & 54.48 & 9.01 & 43.35 & 51.40 \\
\hline 25 & 229 & 28 & 1.0000 & 3 & 3 & 1 & 226.3 & $2.096 \times 10^{-10}$ & $4.771 \times 10^{9}$ & 39.08 & 12.38 & 45.12 & 71.65 \\
\hline 26 & 230 & 28 & 1.0000 & 3 & 3 & 2 & 226.9 & $1.927 \times 10^{-10}$ & $5.190 \times 10^{9}$ & 39.02 & 12.34 & 44.98 & 71.76 \\
\hline 27 & 231 & 28 & .0000 & 3 & 3 & 3 & 227.5 & $1.770 \times 10^{-10}$ & $5.650 \times 10^{9}$ & 38.98 & 12.31 & 44.85 & 71.83 \\
\hline
\end{tabular}

The best Optimality and the best efficiency values for SCCD, RCCD, OCCD and FCCD have been summarized in Tables 13-15 with best optimal combinations. 


\section{Table 13: Optimality Values and Efficiency Values $(\%) \mathrm{k}=4$}

\begin{tabular}{|c|c|c|c|c|c|c|c|c|c|c|c|c|c|c|c|c|c|}
\hline \multirow[t]{3}{*}{$\begin{array}{l} \\
\text { Design } \\
\text { Size }\end{array}$} & $\begin{array}{l}\text { Desig } \\
\mathrm{n} \\
\text { Varia } \\
\text { ble K }\end{array}$ & $\begin{array}{c}\text { Parame } \\
\text { ter } \\
\mathrm{P}\end{array}$ & \multicolumn{5}{|c|}{$\begin{array}{c}\text { Optimal Combination with } \\
\text { corresponding Design Size and } \\
\text { Axial Distance for } \\
\text { A-Optimality Criterion }\end{array}$} & \multicolumn{5}{|c|}{\begin{tabular}{|c} 
Optimal Combination with \\
orresponding Design Size and \\
Axial Distance for \\
D-Optimality Criterion
\end{tabular}} & \multicolumn{5}{|c|}{$\begin{array}{l}\text { Optimal Combination with } \\
\text { corresponding Design Size } \\
\text { and Axial Distance for } \\
\text { G-Optimality Criterion }\end{array}$} \\
\hline & \multirow[b]{3}{*}{4} & \multirow[b]{3}{*}{15} & $\mathrm{~N}$ & $\alpha$ & $r_{f}$ & $r_{\alpha}$ & $n_{C}$ & $\mathrm{~N}$ & $\alpha$ & $r_{f}$ & $r_{\alpha}$ & $n_{C}$ & $\mathrm{~N}$ & $\alpha$ & $r_{f}$ & $r_{\alpha}$ & $n_{C}$ \\
\hline & & & 27 & 2.000 & 1 & 1 & 3 & 51 & 2.000 & 2 & 2 & 3 & 26 & 2.000 & 1 & 1 & 2 \\
\hline SCCD & & & \multicolumn{5}{|c|}{$\begin{array}{l}\text { Aopt value }=28.70(\min ) \\
\text { Aeff value }=52.29(\max )\end{array}$} & \multicolumn{5}{|c|}{$\begin{array}{l}\text { Dopt Value: } \\
\max |\mathrm{M}|=0.0210 \\
\min \left|\mathrm{M}^{-1}\right|=47.72 \\
\text { Deff value }=77.28(\max )\end{array}$} & \multicolumn{5}{|c|}{$\begin{array}{l}\text { Gopt value }=15.17(\min ) \\
\text { Geff value }=98.90(\max )\end{array}$} \\
\hline \multirow{5}{*}{\multicolumn{3}{|c|}{$\begin{array}{l}\text { Best Optimal } \\
\text { Combination for } \\
\text { SCCD }\end{array}$}} & $\mathrm{N}$ & $\alpha$ & $r_{f}$ & $r_{\alpha}$ & $n_{C}$ & $\mathrm{~N}$ & $\alpha$ & $r_{f}$ & $r_{\alpha}$ & $n_{C}$ & $\mathrm{~N}$ & $\alpha$ & $r_{f}$ & $r_{\alpha}$ & $n_{C}$ \\
\hline & & & 27 & 2.000 & 1 & 1 & 3 & 27 & 2.000 & 1 & 1 & 3 & 27 & 2.000 & 1 & 1 & 3 \\
\hline & & & \multirow{2}{*}{\multicolumn{5}{|c|}{ Aopt $=28.70$}} & \multicolumn{5}{|c|}{$D_{o p t}^{\max }=0.0178$} & \multirow{2}{*}{\multicolumn{5}{|c|}{ Gopt $=15.75$}} \\
\hline & & & & & & & & & & & & & & & & & \\
\hline & & & \multicolumn{5}{|c|}{ Aeff $=52.29$} & \multicolumn{5}{|c|}{ Deff $=76.44$} & \multicolumn{5}{|c|}{ Geff $=95.24$} \\
\hline \multirow[b]{3}{*}{$\mathrm{RCCD}$} & \multirow[b]{3}{*}{4} & \multirow[b]{3}{*}{15} & $\mathrm{~N}$ & $\alpha$ & $r_{f}$ & $r_{\alpha}$ & $n_{C}$ & $\mathrm{~N}$ & $\alpha$ & $r_{f}$ & $r_{\alpha}$ & $n_{C}$ & $\mathrm{~N}$ & $\alpha$ & $r_{f}$ & $r_{\alpha}$ & $n_{C}$ \\
\hline & & & 59 & 2.632 & 3 & 1 & 3 & 57 & 2.632 & 3 & 1 & 1 & 26 & 2.000 & 1 & 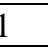 & 2 \\
\hline & & & \multicolumn{5}{|c|}{$\begin{array}{l}\text { Aopt value }=25.17(\min ) \\
\text { Aeff value }=59.60(\max )\end{array}$} & \multicolumn{5}{|c|}{$\begin{array}{l}\text { popt Value: } \\
\max |\mathrm{M}|=0.8071 \\
\operatorname{Min}\left|\mathrm{M}^{-1}\right|=1.2390 \\
\text { Deff value }=98.59(\max )\end{array}$} & $\begin{array}{l}\text { Gopt } \\
\text { Geff v }\end{array}$ & $\begin{array}{l}\text { lue }=15 \\
\text { lue }=98\end{array}$ & $\begin{array}{l}17(\mathrm{n} \\
0(\mathrm{~m}\end{array}$ & $\begin{array}{l}\text { in) } \\
\mathrm{ax})\end{array}$ & \\
\hline & & & $\mathrm{N}$ & $\alpha$ & $r_{f}$ & $r_{\alpha}$ & $n_{C}$ & $\mathrm{~N}$ & $\alpha$ & $r_{f}$ & $r_{\alpha}$ & $n_{C}$ & $\mathrm{~N}$ & $\alpha$ & $r_{f}$ & $r_{\alpha}$ & $n_{C}$ \\
\hline & & & 27 & 2.000 & 1 & 1 & 3 & 27 & 2.000 & 1 & 1 & 3 & 27 & 2.000 & 1 & 1 & 3 \\
\hline Bes & st Optir & mal & & & & & & & $D_{\text {opt }}^{\max }=$ & & & & & & & & \\
\hline Coml & $\begin{array}{l}\text { binatio } \\
\text { RCCD }\end{array}$ & on for & & Aopt $=$ & 8.7 & & & & $D_{\text {opt }}^{\min }=$ & 30. & & & & Gopt $=$ & .75 & & \\
\hline & & & & Aeff $=$ & 52.2 & & & & $\operatorname{Deff}=$ & 76.4 & & & & Geff $=$ & 5.2 & & \\
\hline & & & $\mathrm{N}$ & $\alpha$ & $r_{f}$ & $r_{\alpha}$ & $n_{C}$ & $\mathrm{~N}$ & $\alpha$ & $r_{f}$ & $r_{\alpha}$ & $n_{C}$ & $\mathrm{~N}$ & $\alpha$ & $r_{f}$ & $r_{\alpha}$ & $n_{C}$ \\
\hline & & & 27 & 1.547 & 1 & 1 & 3 & 43 & 1.596 & 2 & 1 & 3 & 73 & 1.366 & & 3 & 1 \\
\hline OCCD & 4 & 15 & Aopt $\mathrm{V}$ & $\begin{array}{l}e=3 \\
e=4\end{array}$ & 85( & & & $\begin{array}{l}\text { Dopt } \\
\max \mid \Lambda \\
\text { Min|N } \\
\text { Deff }\end{array}$ & $\begin{array}{l}\text { lue: } \\
=7.803 \\
=1.282 \\
\text { lue }=6\end{array}$ & $\begin{array}{r}X 10 \\
X 1 \\
2.06\end{array}$ & $\max )$ & & $\begin{array}{l}\text { Gopt } \\
\text { Geff } v\end{array}$ & $\begin{array}{l}\text { ue }=1 \\
\text { de }=9\end{array}$ & $\begin{array}{l}56(\mathrm{n} \\
1(\mathrm{~m}\end{array}$ & & \\
\hline & & & $\mathrm{N}$ & $\alpha$ & $r_{f}$ & $r_{\alpha}$ & $n_{C}$ & $\mathrm{~N}$ & $\alpha$ & $r_{f}$ & $r_{\alpha}$ & $n_{C}$ & $\mathrm{~N}$ & $\alpha$ & $r_{f}$ & $r_{\alpha}$ & $n_{C}$ \\
\hline & & & 27 & 1.547 & 1 & 1 & 3 & 27 & 1.547 & 1 & 1 & 3 & 27 & 1.547 & 1 & 1 & 3 \\
\hline Bes & Optir & & & & & & & & $D_{\text {opt }}^{\max }=$ & & & & & & & & \\
\hline Coml & $\begin{array}{l}\text { binatio } \\
\text { OCCD }\end{array}$ & on for & & Aopt $=$ & 20.1 & & & & $D_{o p t}^{\min }=$ & 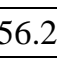 & & & & Gopt $=$ & 57 & & \\
\hline & & & & Aeff $=$ & 52.2 & & & & Deff $=$ & 76.4 & & & & Geff $=$ & 5.2 & & \\
\hline & & & $\mathrm{N}$ & $\alpha$ & $r_{f}$ & $r_{\alpha}$ & $n_{C}$ & $\mathrm{~N}$ & $\alpha$ & $r_{f}$ & $r_{\alpha}$ & $n_{C}$ & $\mathrm{~N}$ & $\alpha$ & $r_{f}$ & $r_{\alpha}$ & $n_{C}$ \\
\hline & & & 41 & 1.000 & 1 & 3 & 1 & 65 & 1.000 & 3 & 2 & 1 & 73 & 1.000 & & $\beta$ & 1 \\
\hline FCCD & 4 & 15 & $\begin{array}{l}\text { Aopt } v \\
\text { Aeff }\end{array}$ & $\begin{array}{l}e=4 \\
e=3\end{array}$ & 12 & $\begin{array}{l}\text { in) } \\
\operatorname{ax}\end{array}$ & & $\begin{array}{l}\text { Dopt } \\
\max \mid \Lambda \\
\text { Min|N } \\
\text { Deff }\end{array}$ & $\begin{array}{l}\text { lue: } \\
=7.049 \\
=1.41 \mathrm{C} \\
\text { lue }=4\end{array}$ & $\begin{array}{r}X 1 \\
5.35 \\
\end{array}$ & $\operatorname{nax})$ & & $\begin{array}{l}\text { Gopt } \\
\text { Geff }\end{array}$ & $\begin{array}{l}\mathrm{e}=1 \\
\mathrm{e}=9\end{array}$ & $6(\mathrm{~m}$ & & \\
\hline & & & $\mathrm{N}$ & $\alpha$ & $r_{f}$ & $r_{\alpha}$ & $n_{C}$ & $\mathrm{~N}$ & $\alpha$ & $r_{f}$ & $r_{\alpha}$ & $n_{C}$ & $\mathrm{~N}$ & $\alpha$ & $r_{f}$ & $r_{\alpha}$ & $n_{C}$ \\
\hline & & & 25 & 1.000 & 1 & 1 & 1 & 25 & 1.000 & 1 & 1 & 1 & 25 & 1.000 & 1 & 1 & 1 \\
\hline $\begin{array}{r}\text { Bes } \\
\text { Coml }\end{array}$ & $\begin{array}{l}\text { St Optir } \\
\text { binatio }\end{array}$ & $\begin{array}{l}\text { mal } \\
\text { on for }\end{array}$ & & Aopt & 58.8 & & & & $\begin{array}{l}\operatorname{lax}^{2 a x}=5 . \\
\min _{p t}=1 .\end{array}$ & $\frac{562}{3572}$ & $\begin{array}{l}10^{-6} \\
10^{5} \\
\end{array}$ & & & Gopt $=$ & 6.48 & & \\
\hline & FCCD & & & Aeff & 25 & & & Deff & 44.52 & & & & & Geff & 1.0 & & \\
\hline
\end{tabular}


African Journal of Mathematics and Statistics Studies

ISSN: 2689-5323

Volume 4, Issue 3, 2021 (pp. 89-117)

www.abjournals.org

Table 14: Optimality Values and Efficiency Values (\%) k = 5

\begin{tabular}{|c|c|c|c|c|c|c|c|c|c|c|c|c|c|c|c|c|c|}
\hline $\begin{array}{l}\text { Desig } \\
\text { n Size }\end{array}$ & $\begin{array}{c}\text { Design } \\
\text { Variabl } \\
\text { e K }\end{array}$ & $\begin{array}{c}\text { Param } \\
\text { eter } \\
\mathrm{P}\end{array}$ & $\begin{array}{r}\text { Opti } \\
\text { corr } \\
\text { an } \\
\text { A }\end{array}$ & $\begin{array}{l}\text { al Com } \\
\text { pondin } \\
\text { Axial L } \\
\text { ptimali }\end{array}$ & $\begin{array}{l}\text { Dinat } \\
\text { Des } \\
\text { istan } \\
\text { y Cri }\end{array}$ & $\begin{array}{l}\text { ion } v \\
\text { gn } S \\
\text { ce fo } \\
\text { terio }\end{array}$ & & $\begin{array}{r}\text { Opt } \\
\text { corr } \\
\text { an } \\
\text { D }\end{array}$ & $\begin{array}{l}\text { nal Com } \\
\text { spondin } \\
\text { Axial D } \\
\text { Optimali }\end{array}$ & $\begin{array}{l}\text { binat } \\
\text { Des } \\
\text { istar } \\
\text { y Cr }\end{array}$ & $\begin{array}{l}\text { on } w \\
\text { ign } S \\
\text { ce fo } \\
\text { terio }\end{array}$ & & $\begin{array}{r}\text { Opti } \\
\text { corr } \\
\text { an } \\
\text { G. }\end{array}$ & $\begin{array}{l}\text { al Com } \\
\text { ponding } \\
\text { Axial L } \\
\text { ptimali }\end{array}$ & $\begin{array}{l}\text { inat } \\
\text { Des } \\
\text { istan } \\
\text { y Cri }\end{array}$ & terio & \\
\hline \multirow[b]{3}{*}{ SCCD } & \multirow{3}{*}{ 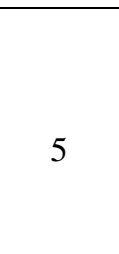 } & \multirow[b]{3}{*}{21} & $\mathrm{~N}$ & $\alpha$ & $r_{f}$ & $r_{\alpha}$ & $n_{C}$ & $\mathrm{~N}$ & $\alpha$ & $r_{f}$ & $r_{\alpha}$ & $n_{C}$ & $\mathrm{~N}$ & $\alpha$ & $r_{f}$ & $r_{\alpha}$ & $n_{C}$ \\
\hline & & & 45 & 2.236 & 1 & 1 & 3 & 44 & 2.236 & 1 & 1 & 2 & 55 & 2.236 & & 2 & 3 \\
\hline & & & \multicolumn{5}{|c|}{$\begin{array}{l}\text { Aopt value }=41.22(\mathrm{~min}) \\
\text { Aeff value }=50.93(\max )\end{array}$} & \multicolumn{5}{|c|}{$\begin{array}{l}\text { Dopt Value: } \\
\max |\mathrm{M}|=0.0119 \\
\text { Min }\left|\mathrm{M}^{-1}\right|=84.28 \\
\text { Deff value }=80.96(\max )\end{array}$} & \multicolumn{5}{|c|}{$\begin{array}{l}\text { Gopt value }=23.53(\min ) \\
\text { Geff value }=89.23(\max )\end{array}$} \\
\hline \multirow{5}{*}{\multicolumn{3}{|c|}{$\begin{array}{l}\text { Best Optimal } \\
\text { Combination for } \\
\text { SCCD }\end{array}$}} & $\mathrm{N}$ & $\alpha$ & $r_{f}$ & $r_{\alpha}$ & $n_{C}$ & $\mathrm{~N}$ & $\alpha$ & $r_{f}$ & $r_{\alpha}$ & $n_{C}$ & $\mathrm{~N}$ & $\alpha$ & $r_{f}$ & $r_{\alpha}$ & $n_{C}$ \\
\hline & & & 45 & 2.236 & 1 & 1 & 3 & 45 & 2.236 & 1 & 1 & 3 & 45 & 2.236 & 1 & 1 & 3 \\
\hline & & & \multirow{2}{*}{\multicolumn{5}{|c|}{ Aopt $=41.22$}} & \multicolumn{5}{|c|}{$D_{\text {opt }}^{\max }=0.0111$} & \multirow{2}{*}{\multicolumn{5}{|c|}{ Gopt $=24.43$}} \\
\hline & & & & & & & & & & & & & & & & & \\
\hline & & & \multicolumn{5}{|c|}{ Aeff $=50.93$} & \multicolumn{5}{|c|}{ Deff $=80.71$} & \multicolumn{5}{|c|}{ Geff $=85.97$} \\
\hline \multirow[b]{3}{*}{$\begin{array}{c}\mathrm{RCC} \\
\mathrm{D}\end{array}$} & \multirow[b]{3}{*}{5} & \multirow[b]{3}{*}{21} & $\mathrm{~N}$ & $\alpha$ & $r_{f}$ & $r_{\alpha}$ & $n_{C}$ & $\mathrm{~N}$ & $\alpha$ & $r_{f}$ & $r_{\alpha}$ & $n_{C}$ & $\mathrm{~N}$ & $\alpha$ & $r_{f}$ & $r_{\alpha}$ & $n_{C}$ \\
\hline & & & 59 & 2.632 & 3 & 1 & 3 & 57 & 2.632 & 3 & 1 & 1 & 26 & 2.000 & & 1 & 2 \\
\hline & & & \multicolumn{5}{|c|}{$\begin{array}{l}\text { Aopt value }=31.57(\min ) \\
\text { Aeff value } 66.53(\max )\end{array}$} & \multicolumn{5}{|c|}{$\begin{array}{l}\text { Dopt Value: } \\
\max |\mathrm{M}|=2.2902 \\
\text { Min }\left|\mathrm{M}^{-1}\right|=0.4366 \\
\text { Deff value }=104.03(\max )\end{array}$} & \multicolumn{5}{|c|}{$\begin{array}{l}\text { Gopt value }=15.17(\min ) \\
\text { Geff value }=98.90(\max )\end{array}$} \\
\hline \multirow{5}{*}{\multicolumn{3}{|c|}{$\begin{array}{l}\text { Best Optimal } \\
\text { Combination for } \\
\text { RCCD }\end{array}$}} & $\mathrm{N}$ & $\alpha$ & $r_{f}$ & $r_{\alpha}$ & $n_{C}$ & $\begin{array}{l}\mathrm{N} \\
\end{array}$ & $\alpha$ & $r_{f}$ & $r_{\alpha}$ & $n_{C}$ & $\mathrm{~N}$ & $\alpha$ & $r_{f}$ & $r_{\alpha}$ & $n_{C}$ \\
\hline & & & 45 & 2.378 & 1 & 1 & 3 & 45 & 2.378 & 1 & 1 & 3 & 45 & 2.378 & 1 & 1 & 3 \\
\hline & & & \multirow{2}{*}{\multicolumn{5}{|c|}{ Aopt $=39.37$}} & & $D_{o p t}^{\max }=$ & 0.03 & & & & & & & \\
\hline & & & & & & & & & $\overline{D_{\text {opt }}^{\min }=}$ & 25.9 & & & & Gopt $=$ & 25.9 & & \\
\hline & & & & Aeff $=$ & 53.3 & & & & Deff & $=76$. & & & & Geff $=$ & 83.0 & & \\
\hline & & & $\mathrm{N}$ & $\alpha$ & $r_{f}$ & $r_{\alpha}$ & $n_{C}$ & $\mathrm{~N}$ & $\alpha$ & $r_{f}$ & $r_{\alpha}$ & $n_{C}$ & $\mathrm{~N}$ & $\alpha$ & $r_{f}$ & $r_{\alpha}$ & $n_{C}$ \\
\hline & & & 55 & 1.577 & 1 & 2 & 3 & 45 & 1.724 & 1 & 1 & 3 & 95 & 1.526 & & 3 & 1 \\
\hline $\begin{array}{c}\mathrm{OCC} \\
\mathrm{D}\end{array}$ & 5 & 21 & $\begin{array}{l}\text { Aopt } \\
\text { Aeff }\end{array}$ & $\begin{array}{l}e=4 \\
\mathrm{e}=4\end{array}$ & .31( & $\begin{array}{l}\min ) \\
\max \end{array}$ & & $\begin{array}{l}\text { Dopt } \\
\max \mid \Lambda \\
\text { Min|N } \\
\text { Deff }\end{array}$ & $\begin{array}{l}\text { alue: } \\
=1.317 \\
=1.59 \\
\text { alue }=6\end{array}$ & $\begin{array}{l}X 10 \\
5 \times 1 \\
5.36\end{array}$ & $\begin{array}{l}3^{3} \\
\max \end{array}$ & & $\begin{array}{l}\text { Gopt } \\
\text { Geff }\end{array}$ & $\begin{array}{l}\text { lue }=22 \\
\text { lue }=93\end{array}$ & $\begin{array}{l}40(1 \\
73(n\end{array}$ & & \\
\hline & & & $\mathrm{N}$ & $\alpha$ & $r_{f}$ & $r_{\alpha}$ & $n_{C}$ & $\mathrm{~N}$ & $\alpha$ & $r_{f}$ & $r_{\alpha}$ & $n_{C}$ & $\mathrm{~N}$ & $\alpha$ & $r_{f}$ & $r_{\alpha}$ & $n_{C}$ \\
\hline & est Optin & & 45 & 1.724 & 1 & 1 & 3 & 45 & 1.724 & 1 & 1 & 3 & 45 & 1.724 & 1 & 1 & 3 \\
\hline Con & nbinatiol & & & & & & & & $\max _{\text {opt }}=1$ & 317 & $10^{-}$ & & & & & & \\
\hline & OCCD & & & Aopt $=$ & 44.7 & & & & $\frac{\min _{o p t}=7 .}{\text { int }}$ & 595 & $10^{3}$ & & & Gopt $=$ & 23.3 & & \\
\hline & & & & Aeff $=$ & 49.1 & & & & Deff $=$ & 65.3 & & & & Geff $=$ & 90.0 & & \\
\hline & & & $\mathrm{N}$ & $\alpha$ & $r_{f}$ & $r_{\alpha}$ & $n_{C}$ & $\mathrm{~N}$ & $\alpha$ & $r_{f}$ & $r_{\alpha}$ & $n_{C}$ & $\mathrm{~N}$ & $\alpha$ & $r_{f}$ & $r_{\alpha}$ & $n_{C}$ \\
\hline & & & 63 & 1.000 & 1 & 3 & 1 & 127 & 1.000 & 3 & 3 & 1 & 86 & 1.000 & & 2 & 2 \\
\hline FCCD & 5 & 21 & $\begin{array}{l}\text { Aopt } \\
\text { Aeff }\end{array}$ & $\begin{array}{l}\mathrm{e}=7 \\
\mathrm{e}=2\end{array}$ & $\begin{array}{l}4.43 \\
3.21(\end{array}$ & $\begin{array}{l}\min \\
\max \end{array}$ & & $\begin{array}{l}\text { Dopt } \\
\max \mid \Lambda \\
\text { Min|N } \\
\text { Deff }\end{array}$ & $\begin{array}{l}\text { alue: } \\
=1.640 \\
=1 \mid=1.64 \\
\text { alue }=4\end{array}$ & $\begin{array}{l}\mathrm{X} 10 \\
\mathrm{X} 1 \\
5.34\end{array}$ & $\max$ & & $\begin{array}{l}\text { Gopt } \\
\text { Geff }\end{array}$ & $\begin{array}{l}e=2 \\
e=9\end{array}$ & $\begin{array}{l}19(1 \\
66(n\end{array}$ & & \\
\hline & & & $\mathrm{N}$ & $\alpha$ & $r_{f}$ & $r_{\alpha}$ & $n_{C}$ & $\mathrm{~N}$ & $\alpha$ & $r_{f}$ & $r_{\alpha}$ & $n_{C}$ & $\mathrm{~N}$ & $\alpha$ & $r_{f}$ & $r_{\alpha}$ & $n_{C}$ \\
\hline & & & 53 & 1.000 & 1 & 2 & 1 & 53 & 1.000 & 1 & 2 & 1 & 53 & 1.000 & 1 & 2 & 1 \\
\hline & & & & & & & & & $\max _{\text {opt }}=2$ & 379 & $10^{-}$ & & & & & & \\
\hline $\begin{array}{l}\text { Be } \\
\text { Con }\end{array}$ & $\begin{array}{l}\text { est Optin } \\
\text { nbinatiol }\end{array}$ & nal & & Aopt & 2.1 & & & & $\min =4$. & 205 & $10^{7}$ & & & Gopt & 55 & & \\
\hline & FC & & & Aeff & $20 .$. & & & & J) & & & & & Gef & 02.1 & & \\
\hline
\end{tabular}


African Journal of Mathematics and Statistics Studies

ISSN: 2689-5323

Volume 4, Issue 3, 2021 (pp. 89-117)

www.abjournals.org

\section{Table 15: Optimality Values and Efficiency Values (\%) K = 6}

\begin{tabular}{|c|c|c|c|c|c|c|c|c|c|c|c|c|c|c|c|c|c|}
\hline $\begin{array}{l}\text { Desi } \\
\text { gn } \\
\text { Size }\end{array}$ & $\begin{array}{c}\text { Design } \\
\text { Variab } \\
\text { le K }\end{array}$ & $\begin{array}{c}\text { Para } \\
\text { meter } \\
\mathrm{P}\end{array}$ & $\begin{array}{r}\text { Opti } \\
\text { corr } \\
\text { an } \\
\mathrm{A}\end{array}$ & $\begin{array}{l}\text { al Con } \\
\text { spondin } \\
\text { Axial I } \\
\text { Optimal }\end{array}$ & $\mathrm{y}_{1}$ & terio & & $\begin{array}{l}\text { Opti } \\
\text { corr } \\
\text { an } \\
\text { D- }\end{array}$ & $\begin{array}{l}\text { nal Con } \\
\text { spondin } \\
\text { I Axial } \\
\text { Optimal }\end{array}$ & $\mathrm{y}_{\mathrm{C}}$ & $\begin{array}{l}\text { lon } \mathrm{W} \\
\text { ign } \mathrm{S} \\
\text { ce fo } \\
\text { terio }\end{array}$ & & & $\begin{array}{l}\text { al Com } \\
\text { pondin } \\
\text { Axial D } \\
\text { ptimali }\end{array}$ & $\begin{array}{l}\text { Des } \\
\text { istan } \\
\text { y } \mathrm{Cr}\end{array}$ & teric & \\
\hline \multirow[b]{3}{*}{$\begin{array}{c}\text { SCC } \\
\text { D }\end{array}$} & \multirow{3}{*}{ 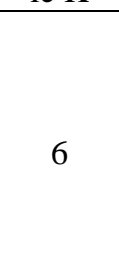 } & \multirow[b]{3}{*}{28} & $\mathrm{~N}$ & $\alpha$ & $r_{f}$ & $r_{\alpha}$ & $n_{C}$ & $\mathrm{~N}$ & $\alpha$ & $r_{f}$ & $r_{\alpha}$ & $n_{C}$ & $\mathrm{~N}$ & $\alpha$ & $r_{f}$ & $r_{\alpha}$ & $n_{C}$ \\
\hline & & & 79 & 2.450 & 1 & 1 & 3 & 91 & 2.236 & 1 & 2 & 3 & 91 & 2.450 & 1 & 2 & 3 \\
\hline & & & \multicolumn{5}{|c|}{$\begin{array}{l}\text { Aopt value }=61.13(\mathrm{~min}) \\
\text { Aeff value }=45.80(\mathrm{max})\end{array}$} & \multicolumn{5}{|c|}{$\begin{array}{l}\text { Dopt Value: } \\
\max |\mathrm{M}|=0.0079 \\
\operatorname{Min}\left|\mathrm{M}^{-1}\right|=126.39 \\
\text { Deff value }=84.13(\max )\end{array}$} & \multicolumn{5}{|c|}{$\begin{array}{l}\text { Gopt value }=30.33(\mathrm{~min}) \\
\text { Geff value }=92.31(\mathrm{max})\end{array}$} \\
\hline \multirow{5}{*}{\multicolumn{3}{|c|}{$\begin{array}{l}\text { Best Optimal } \\
\text { Combination for } \\
\text { SCCD }\end{array}$}} & $\mathrm{N}$ & $\alpha$ & $r_{f}$ & $r_{\alpha}$ & $n_{C}$ & $\mathrm{~N}$ & $\alpha$ & $r_{f}$ & $r_{\alpha}$ & $n_{C}$ & $\mathrm{~N}$ & $\alpha$ & $r_{f}$ & $r_{\alpha}$ & $n_{C}$ \\
\hline & & & 91 & 2.450 & 1 & 2 & 3 & 91 & 2.450 & 1 & 2 & 3 & 91 & 2.450 & 1 & 2 & 3 \\
\hline & & & \multirow{2}{*}{\multicolumn{5}{|c|}{ Aopt $=66.25$}} & \multicolumn{5}{|c|}{$D_{\text {opt }}^{\max }=0.0078$} & \multirow{2}{*}{\multicolumn{5}{|c|}{ Gopt $=24.43$}} \\
\hline & & & & & & & & & $D_{o p t}^{\min }=$ & 126 & & & & & & & \\
\hline & & & \multicolumn{5}{|c|}{ Aeff $=42.26$} & \multicolumn{5}{|c|}{ Deff $=84.13$} & \multicolumn{5}{|c|}{ Geff $=85.97$} \\
\hline \multirow[b]{3}{*}{$\begin{array}{c}\mathrm{RCC} \\
\mathrm{D}\end{array}$} & \multirow[b]{3}{*}{6} & \multirow[b]{3}{*}{28} & $\mathrm{~N}$ & $\alpha$ & $r_{f}$ & $r_{\alpha}$ & $n_{C}$ & $\mathrm{~N}$ & $\alpha$ & $r_{f}$ & $r_{\alpha}$ & $n_{C}$ & $\mathrm{~N}$ & $\alpha$ & $r_{f}$ & $r_{\alpha}$ & $n_{C}$ \\
\hline & & & 207 & 3.722 & 3 & 1 & 3 & 205 & 3.722 & 3 & 1 & 1 & 91 & 2.378 & 1 & 2 & 3 \\
\hline & & & \multicolumn{5}{|c|}{$\begin{array}{l}\text { Aopt value }=37.27(\min ) \\
\text { Aeff value }=75.14(\max )\end{array}$} & \multicolumn{5}{|c|}{$\begin{array}{l}\text { Dopt Value: } \\
\max |\mathrm{M}|=7.8319 \\
\text { Min }\left|\mathrm{M}^{-1}\right|=0.1277 \\
\text { Deff value }=107.64(\max )\end{array}$} & \multicolumn{5}{|c|}{$\begin{array}{l}\text { Gopt value }=29.75(\min ) \\
\text { Geff value }=94.12(\max )\end{array}$} \\
\hline \multirow{5}{*}{\multicolumn{3}{|c|}{$\begin{array}{l}\text { Best Optimal } \\
\text { Combination for } \\
\text { RCCD }\end{array}$}} & $\mathrm{N}$ & $\alpha$ & $r_{f}$ & $r_{\alpha}$ & $n_{C}$ & $\mathrm{~N}$ & $\alpha$ & $r_{f}$ & $r_{\alpha}$ & $n_{C}$ & $\mathrm{~N}$ & $\alpha$ & $r_{f}$ & $r_{\alpha}$ & $n_{C}$ \\
\hline & & & 91 & 2.378 & 1 & 2 & 3 & 91 & 2.378 & 1 & 2 & 3 & 91 & 2.378 & 1 & 2 & 3 \\
\hline & & & \multirow{2}{*}{\multicolumn{5}{|c|}{ Aopt $=66.22$}} & & $\bar{D}_{\text {opt }}^{\max }=$ & $\overline{0.00}$ & & & & & & & \\
\hline & & & & & & & & & $D_{o p t}^{\min }=$ & 44 & & & & Gopt $=$ & 29.7 & & \\
\hline & & & & Aeff & 42 . & & & & Deff & 82 & & & & Geff $=$ & 94.1 & & \\
\hline & & & $\mathrm{N}$ & $\alpha$ & $r_{f}$ & $r_{\alpha}$ & $n_{C}$ & $\mathrm{~N}$ & $\alpha$ & $r_{f}$ & $r_{\alpha}$ & $n_{C}$ & $\mathrm{~N}$ & $\alpha$ & $r_{f}$ & $r_{\alpha}$ & $n_{C}$ \\
\hline & & & 27 & 1.547 & 1 & 1 & 3 & 43 & 1.596 & 2 & 1 & 3 & 73 & 1.366 & 3 & 3 & 1 \\
\hline $\begin{array}{c}\text { OCC } \\
\text { D }\end{array}$ & 6 & 28 & $\begin{array}{l}\text { Aopt } \\
\text { Aeff }\end{array}$ & $\begin{array}{l}\text { lue }= \\
\text { lue }=\end{array}$ & .0 & nin & & $\begin{array}{l}\text { Dopt } \\
\max \mid 1 \\
\text { Min|1 } \\
\text { Deff }\end{array}$ & 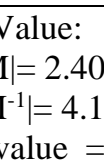 & $\lambda$ & $\begin{array}{l}-5 \\
10^{4} \\
(\mathrm{ma}\end{array}$ & & $\begin{array}{l}\text { Gopt } \\
\text { Geff }\end{array}$ & $\begin{array}{l}\text { alue }=2 \\
\text { alue }=9\end{array}$ & 9.31 & $\begin{array}{l}\min \\
\max \end{array}$ & \\
\hline & & & $\mathrm{N}$ & $\alpha$ & $r_{f}$ & $r_{\alpha}$ & $n_{C}$ & $\mathrm{~N}$ & $\alpha$ & $\overline{r_{f}}$ & $r_{\alpha}$ & $n_{C}$ & $\mathrm{~N}$ & $\alpha$ & $r_{f}$ & $r_{\alpha}$ & $n_{C}$ \\
\hline & & & 91 & 1.755 & 1 & 1 & 3 & 91 & 1.755 & 1 & 1 & 3 & 91 & 1.755 & 1 & 1 & 3 \\
\hline & est Optir & & & & & & & & $p t=9$ & & $10^{-6}$ & & & & & & \\
\hline & mbinatio & & & Aopt & 4. & & & & $\min _{p p t}=1$ & 974 & $10^{5}$ & & & Gopt & 29.8 & & \\
\hline & & & & Aeff & 51. & & & & Deff & 66 & & & & Geff $=$ & 93.7 & & \\
\hline & & & $\mathrm{N}$ & $\alpha$ & $r_{f}$ & $r_{\alpha}$ & $n_{C}$ & $\mathrm{~N}$ & $\alpha$ & $r_{f}$ & $r_{\alpha}$ & $n_{C}$ & $\mathrm{~N}$ & $\alpha$ & $r_{f}$ & $r_{\alpha}$ & $n_{C}$ \\
\hline & & & 41 & 1.000 & 1 & 3 & 1 & 65 & 1.000 & 3 & 2 & 1 & 73 & 1.000 & 3 & 3 & 1 \\
\hline $\begin{array}{c}\text { FCC } \\
\text { D }\end{array}$ & 6 & 28 & $\begin{array}{l}\text { Aopt } \\
\text { Aeff }\end{array}$ & $\begin{array}{l}\text { lue }= \\
\text { lue }=\end{array}$ & 321 & mi & & $\begin{array}{l}\text { Dopt } \\
\max \mid 1 \\
\text { Min|1 } \\
\text { Deff }\end{array}$ & $\begin{array}{l}\text { Value: } \\
\mid \begin{array}{l}\mid=2.96 \\
{ }^{-1} \mid=3.3 \\
\text { value }=\end{array}\end{array}$ & 45.6 & $\begin{array}{l}{ }^{-10} \\
10^{9} \\
(\max \\
\end{array}$ & & $\begin{array}{l}\text { Gopt } \\
\text { Geff }\end{array}$ & $\begin{array}{l}\text { alue }=2 \\
\text { lue }=9\end{array}$ & 3.56 & $\begin{array}{l}\min \\
\max \end{array}$ & \\
\hline & & & $\mathrm{N}$ & $\alpha$ & $r_{f}$ & $r_{\alpha}$ & $n_{C}$ & $\mathrm{~N}$ & $\alpha$ & $r_{f}$ & $r_{\alpha}$ & $n_{C}$ & $\mathrm{~N}$ & $\alpha$ & $r_{f}$ & $r_{\alpha}$ & $n_{C}$ \\
\hline & & & 89 & 1.000 & 1 & 2 & 1 & 89 & 1.000 & 1 & 2 & 1 & 89 & 1.000 & 1 & 2 & 1 \\
\hline & & & & & & & & & $\operatorname{mox}^{\circ a x}=2$ & 223 & K $10^{-}$ & & & & & & \\
\hline & $\begin{array}{l}\text { est Opt11 } \\
\text { mbinatio }\end{array}$ & & & Aopt & $1+0$ & & & & $\min =4$ & $\overline{00}$ & $10^{9}$ & & & Gop & 301 & & \\
\hline & $\mathrm{FCCl}$ & & & Aeff & 19. & & & & Deff & 4. & & & & Geff $=$ & 93.0 & & \\
\hline
\end{tabular}




\section{DISCUSSION OF RESULTS}

With reference to Table1, for SCCD full factorial replicates, the optimal combination of [1:1:3] yielded the best $A_{\text {opt }}$ value of 28.70 with A-efficiency value of $52.29 \%$. The associated design is of size $\mathrm{N}=27$ and contains one complete $2^{4}$ factorial points, one complete $2 \mathrm{k}$ axial points and three center points. Optimal combination of [1:1:2] yielded the best $\mathrm{G}_{\text {opt }}$ value of 15.17 with the best G-efficiency value of $98.90 \%$. The associated design is of size $\mathrm{N}=26$ and contains one complete $2^{4}$ factorial points, one complete $2 \mathrm{k}$ axial points and two center points. It is interesting to note that the design associated with the [1:1:2] combination resulted in the second best A-Optimal value of 33.05 as well as second best A-efficiency value of $45.40 \%$ Optimal combination of [2:2:3] produced the best D-efficiency value of $77.28 \%$. The associated design is of size $\mathrm{N}=51$. It is necessary to note that the design associated with [1:1:2] resulted in the second best D-efficiency value and exhibits a smaller design size of $\mathrm{N}=26$ and has a run size efficiency advantage over the combination of [2:2:3] with $\mathrm{N}=51$. The optimal combination of [1:1:3] yielded the most efficient design with design size of $\mathrm{N}=27$, and also has a run size efficiency advantage over other combinations. Similarly, the combination of [1:1:3] producing best $A_{\text {opt }}$ value of 28.70 and best A-efficiency value of $52.29 \%$ was also very good in terms of $\mathrm{G}_{\text {opt }}$ and G-efficiency value. Precisely, the combination of [1:1:3] produced the second best $\mathrm{G}_{\mathrm{opt}}$ value of 15.75 and G-efficiency value of $95.24 \%$. The optimal combinations of [1:1:2] and [2:2:3] produced best $D_{\text {opt }}^{\max }$ values of 0.0209 and 0.0210 , respectively. It is important to observe that the slight difference in these values is due to round-up error. The corresponding G-efficiency values are $77.27 \%$ and $77.28 \%$, respectively. Of the two D-Optimal combinations, the combination [1:1:2], exhibits run size efficiency having a smaller design size of 26 against the combination [2:2:3] with $\mathrm{N}=51$. [1:1:3] yielded the best optimal combination and can be considered the most efficient design

Similarly, from Table 2, for SCCD full factorial replicates, the optimal combination of [1:1:3] yielded the best $A_{\text {opt }}$ value of 41.22 with A-efficiency value of $50.93 \%$. The associated design is of size $\mathrm{N}=45$ and contains one complete $2^{5}$ factorial points, one complete $2 \mathrm{k}$ axial points and three center points. Optimal combination of [1:2:3] yielded the best $\mathrm{G}_{\text {opt }}$ value of 23.53 with the best G-efficiency value of $89.23 \%$. The associated design is of size $\mathrm{N}=55$ and contains one complete $2^{5}$ factorial points, two complete $2 \mathrm{k}$ axial points and three center points. Optimal combination of [1:1:2] yielded the best $D_{o p t}^{\max }$ value of 0.0119 with the best D-efficiency value of $80.96 \%$. The associated design is of size $\mathrm{N}=44$ and contains one complete $2^{5}$ factorial points, one complete $2 \mathrm{k}$ axial points and two center points. Optimal combination of [1:1:2] yielded an Aopt value of 49.10 and A-efficiency value of $42.75 \%$, best $D_{o p t}^{\max }$ value of 0.0119

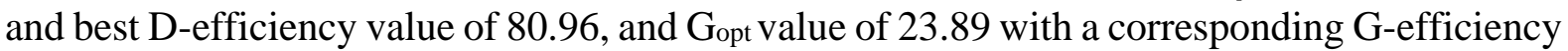
value of $87.92 \%$. The associated design size is $N=44$. Optimal combination of [1:1:3] yielded best $A_{\text {opt }}$ value of 41.22 and best A-efficiency value of 50.93\%, a $D_{o p t}^{\max }$ value of 0.0111 and D-efficiency value of 80.71 and $\mathrm{G}_{\text {opt }}$ value of 23.89 with a corresponding G-efficiency value of $87.92 \%$. The associated design size is $\mathrm{N}=45$. Optimal combination of [1:2:3] yielded the second best $\mathrm{A}_{\mathrm{opt}}$

From Table 3, for SCCD full factorial replicates, the optimal combination of [1:1:3] yielded the best Aopt value of 61.13 with A-efficiency value of $45.80 \%$. The associated design is of size $\mathrm{N}=79$ and contains one complete $2^{6}$ factorial points, one complete $2 \mathrm{k}$ axial points and three center points. Optimal combination of [1:2:3] yielded the best $\mathrm{G}_{\text {opt }}$ value of 30.33 with the best G-efficiency value of $92.31 \%$, and also the best $D_{o p t}^{\max }$ value of 126.39 with corresponding best 
D-efficiency value of $84.13 \%$. The associated design is of size $\mathrm{N}=91$ and contains one complete $2^{6}$ factorial points, two complete $2 \mathrm{k}$ axial points and three center points. Notice that the design associated with [1:1:3] recorded the best A-efficiency value of $45.80 \%$ with corresponding D and G-efficiency values of $82.54 \%$ and $69.66 \%$, respectively. The design associated with [1:2:3] yielded the second best A-efficiency value of $42.26 \%$ with corresponding best D and G-efficiency values of $84.13 \%$ and $92.31 \%$, respectively. Though, with larger design size of $\mathrm{N}=91$ and G-efficiency value of $92.31 \%$, the [1:2:3] combination exhibits a comparative advantage over the [1:1:3] combination with smaller design size of $\mathrm{N}=$ 79 and G-efficiency value of $69.66 \%$.

From Table 4, for RCCD full factorial replicates, the optimal combination of [3:1:3] yielded the best Aopt value of 25.17 with A-efficiency value of $59.60 \%$. The associated design is of size $\mathrm{N}=59$ and contains three complete $2^{4}$ factorial points, one complete $2 \mathrm{k}$ axial points and three center points. Optimal combination of [1:1:2] yielded the best $\mathrm{G}_{\text {opt }}$ value of 15.17 with the best G-efficiency value of $98.90 \%$. The associated design is of size $\mathrm{N}=26$ and contains one complete $2^{4}$ factorial points, one complete $2 \mathrm{k}$ axial points and two center points. Optimal combination of [3:1:1] yielded the best $D_{\text {opt }}^{\max }$ value of 0.0807 with the best D-efficiency value of $98.59 \%$. The associated design is of size $\mathrm{N}=57$ and contains three complete $2^{4}$ factorial points, one complete $2 \mathrm{k}$ axial points and one center point. Observe that the designs associated with best $\mathrm{A}$ and $\mathrm{D}$ criteria produced G-efficiency values below $50 \%$ of the maximum Gefficiency value. Optimal combination of [1:1:3] recorded best A, D and G-efficiency values with run-size efficiency advantage over other combinations.

From Table 5, for RCCD full factorial replication, the optimal combination of [3:1:3] yielded the best Aopt value of 31.57 with A-efficiency value of $66.53 \%$. The associated design is of size $\mathrm{N}=109$ and contains three complete $2^{5}$ factorial points, one complete $2 \mathrm{k}$ axial points and three center points. Optimal combination of [1:2:2] yielded the best $\mathrm{G}_{\text {opt }}$ value of 23.81 with the best G-efficiency value of $88.19 \%$. The associated design is of size $\mathrm{N}=54$ and contains one complete $2^{5}$ factorial points, two complete $2 \mathrm{k}$ axial points and two center points. Optimal combination of [3:1:1] yielded the best $D_{\text {opt }}^{\max }$ value of 2.2902 with super D-efficiency value of $104.03 \%$. The associated design is of size $\mathrm{N}=107$ and contains three complete $2^{5}$ factorial points, one complete $2 \mathrm{k}$ axial points and one center point. Optimal combinations of [3:1:1], [3:1:2] and [3:1:3], yielded best $D_{o p t}^{\max }$ values of 2.2902, 2.1325, and 1.9621, respectively and corresponding super D-efficiency values of $104.03 \%, 103.68 \%$ and $103.27 \%$ respectively and large A-efficiency values of $61.10 \%, 64.05$ and $66.53 \%$, respectively with design sizes of $\mathrm{N}=$

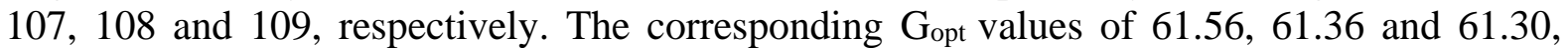
respectively with G-efficiency values of $34.11 \%, 34.23 \%$ and $34.26 \%$, respectively recorded less than $50 \%$ of the maximum G-efficiency value of $88.19 \%$. Comparatively, the design associated with [1:1:3] seems to yield the best optimal combination with a smaller design size of $\mathrm{N}=45$.

From Table 6, for RCCD full factorial replicates, the optimal combination of [3:1:3] yielded the best $A_{\text {opt }}$ value of 37.27 with A-efficiency value of $75.14 \%$. The associated design is of size $\mathrm{N}=207$ and contains three complete $2^{6}$ factorial points, one complete $2 \mathrm{k}$ axial points and three center points. Optimal combination of [1:2:3] yielded the best $\mathrm{G}_{\text {opt }}$ value of 29.75 with the best G-efficiency value of $94.12 \%$. The associated design is of size $\mathrm{N}=91$ and contains one complete $2^{6}$ factorial points, two complete $2 \mathrm{k}$ axial points and three center points. Optimal combination of [3:1:3] resulted in best $D_{o p t}^{\max }$ value of 7.8319 with super D-efficiency value of 
$107.64 \%$. It is worthy to note that the designs associated with [2:1:1], [2:1:2], [2:1:3], [3:1:1], [3:1:2], [3:1:3], [3:2:1], [3:2:2], and [3:2:3], recorded $D_{\text {opt }}^{\max }$ values of 2.8628, 2.6400, 2.4083, $7.8319,7.2505,6.6946,1.0821,1.0673$ and 1.0409 , respectively with corresponding super Defficiency values of $103.82 \%, 103.52 \%, 103.18 \%, 107.64 \%, 107.30 \%, 107.0 \%, 100.30 \%$, $100.20 \%$ and $100.20 \%$ respectively and corresponding large A-efficiency values of $62.50 \%$, $65.24 \%, 67.55 \%, 74.06 \%, 75.14 \%, 50.39 \%, 50.39 \%$ and $55.84 \%$ respectively. Their corresponding G-efficiency values of $35.00 \%, 35.13 \%, 35.19 \%, 24.42 \%, 24.45 \%, 24.47 \%$, $44.71 \%, 44.97 \%$ and $45.15 \%$, respectively are all less than $50 \%$ of the best G-efficiency value of $94.12 \%$ and as such cannot be considered a good optimal combination. The design associated with [1:2:3] tends to be comparatively better than other combinations.

Similarly, from Table 7 for OCCD full factorial replicates, the optimal combination of [1:1:3] yielded the best Aopt value of 31.35 with A-efficiency value of $47.85 \%$. The associated design is of size $\mathrm{N}=27$ and contains one complete $2^{4}$ factorial points, one complete $2 \mathrm{k}$ axial points and three center points. Optimal combination of [3:3:1] yielded the best $\mathrm{G}_{\text {opt value of } 15.56}$ with the best G-efficiency value of $96.41 \%$. The associated design is of size $\mathrm{N}=73$ and contains three complete $2^{4}$ factorial points, three complete $2 \mathrm{k}$ axial points and one center point. Optimal combination of [2:1:3] yielded the best $D_{o p t}^{\max }$ value of $7.803 \times 10^{-4}$ with the best D-efficiency value of $62.06 \%$. The associated design is of size $\mathrm{N}=43$ and contains two complete $2^{4}$ factorial points, one complete $2 \mathrm{k}$ axial points and three center points. Optimal combination of [1:1:3] yielded the best $A_{\text {opt }}$ value of 31.35 with A-efficiency value of $47.85 \%$. The associated design size is $\mathrm{N}=27$ with a corresponding $D_{o p t}^{\max }$ value of $4.924 \times 10^{-4}$ and D-efficiency value of $60.18 \%$, exhibits a run-size efficiency advantage over the maximum D-efficiency value of $62.06 \%$ with associated design size of $\mathrm{N}=43$. The corresponding $\mathrm{G}_{\text {opt }}$ value of 16.82 and Gefficiency value of $90.07 \%$ also exhibits a run-size efficiency advantage over the maximum Gefficiency value of $96.41 \%$ with a design size of $\mathrm{N}=73$. Therefore, the optimal combination of [1:1:3] tends to yield better efficient design compared to other combinations

From Table 8, for OCCD full factorial replication, the optimal combination of [1:2:3] yielded the best $A_{\text {opt }}$ value of 42.31 with A-efficiency value of $49.64 \%$. The associated design is of size $\mathrm{N}=55$ and contains one complete $2^{5}$ factorial points, two complete $2 \mathrm{k}$ axial points and three center points. Optimal combination of [2:3:1] yielded the best $\mathrm{G}_{\text {opt }}$ value of 22.40 with the best G-efficiency value of $93.73 \%$. The associated design is of size $\mathrm{N}=95$ and contains two complete $2^{5}$ factorial points, three complete $2 \mathrm{k}$ axial points and one center point. Optimal combination of [1:1:3] yielded the best $D_{\text {opt }}^{\max }$ value of $1.317 \mathrm{X} 10^{-4}$ with best $\mathrm{D}$-efficiency value of $65.36 \%$. The associated design is of size $\mathrm{N}=45$ and contains one complete $2^{5}$ factorial points, one complete $2 \mathrm{k}$ axial points and three center points. Comparatively, the optimal combination of $[1: 1: 3]$ yielded the best optimal combination.

From Table 9, for OCCD full factorial replicates, the optimal combination of [1:3:3] yielded the best $A_{\text {opt }}$ value of 53.09 with A-efficiency value of $52.74 \%$. The associated design is of size $\mathrm{N}=103$ and contains one complete $2^{6}$ factorial points, three complete $2 \mathrm{k}$ axial points and three

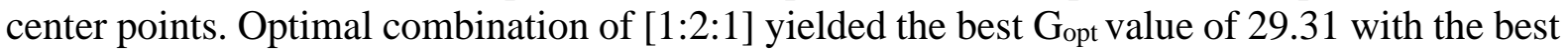
G-efficiency value of $95.53 \%$. The associated design is of size $\mathrm{N}=89$ and contains one complete $2^{6}$ factorial points, two complete $2 \mathrm{k}$ axial points and one center point. Optimal combination of [1:1:3] yielded the best $D_{\text {opt }}^{\max }$ value of $2.408 \times 10^{-5}$ with D-efficiency value of $68.40 \%$. The associated design is of size $\mathrm{N}=79$ and contains one complete $2^{6}$ factorial points, 
one complete $2 \mathrm{k}$ axial point and three center points. Comparatively, the optimal combination of [1:1:3] also yielded the best efficient design.

Similarly, from Table 10, for FCCD full factorial replicates, the optimal combination of [1:3:1] yielded the best $\mathrm{A}_{\text {opt }}$ value of 48.20 with A-efficiency value of $31.12 \%$. The associated design is of size $\mathrm{N}=41$ and contains one complete $2^{4}$ factorial points, three complete $2 \mathrm{k}$ axial points and one center point. Optimal combination of [3:3:1] yielded the best $G_{\text {opt }}$ value of 16.05 with the best G-efficiency value of $93.46 \%$. The associated design is of size $\mathrm{N}=73$ and contains three complete $2^{4}$ factorial points, three complete $2 \mathrm{k}$ axial points and one center point. Optimal combination of [3:2:1] yielded the best $D_{\text {opt }}^{\max }$ value of $7.049 \times 10^{-6}$ with the best D-efficiency value of $45.35 \%$. The associated design is of size $\mathrm{N}=65$ and contains three complete $2^{4}$ factorial points, two complete $2 \mathrm{k}$ axial points and one center point. Observe that optimal combinations of [1:1:1], [2:2:2] and [3:3:3] yielded the same $A_{o p t}, D_{o p t}$, and $G_{o p t}$ values. Their corresponding A, D and G-efficiencies, also yielded the same values, but different design sizes of $\mathrm{N}=25,50$ and 75, respectively. Though the optimality and efficiency values are the same, the [1:1:1] combination is preferred over the [2:2:2] and [3:3:3] because it has the smallest design size efficiency value, and therefore can be considered the best optimal and efficient combination.

From Table 11, for FCCD full factorial replication, the optimal combination of [1:3:1] yielded the best $A_{\text {opt }}$ value of 74.43 with A-efficiency value of $28.21 \%$. The associated design is of size $\mathrm{N}=63$ and contains one complete $2^{5}$ factorial points, three complete $2 \mathrm{k}$ axial points and one center point. Optimal combination of [3:3:1] yielded the best $D_{o p t}^{\max }$ value of $6.100 \times 10^{-8}$ with best D-efficiency value of 45.34\%. Observe that optimal combinations of [1:1:1], [2:2:2] and [3:3:3] yielded the same best $G_{\text {opt }}$ values of 22.19 and G-efficiency values of $94.66 \%$, respectively. The design associated with [1:1:1] combination is preferred over the [2:2:2] and [3:3:3] because it has the smallest design size efficiency value of $\mathrm{N}=43$ against the combination of [2:2:2] and [3:3:3] with design sizes of $\mathrm{N}=86$ and 128, respectively. Comparatively, the design associated with [1:2:1], yielded the best optimal combination.

Finally, from Table 12, for FCCD full factorial replicates, the optimal combination of [1:3:1] yielded the best $A_{\text {opt }}$ value of 121.50 with A-efficiency value of $23.05 \%$. The associated design is of size $\mathrm{N}=101$ and contains one complete $2^{6}$ factorial points, three complete $2 \mathrm{k}$ axial points and one center point. Optimal combination of [2:3:1] yielded the best $\mathrm{G}_{\text {opt }}$ value of 28.56 with the best G-efficiency value of $98.06 \%$ as well as best $D_{o p t}^{\max }$ value of $2.968 \times 10^{-10}$, with best D-efficiency value of $45.68 \%$ The associated design is of size $\mathrm{N}=165$ and contains two complete $2^{6}$ factorial points, three complete $2 \mathrm{k}$ axial points and one center point. Comparatively, the design associated with [1:2:1] tends to yield the best optimal combination.

It is observed that, A-Optimal design satisfies the criterion $A=\operatorname{mintrace}\left[\left(X^{T} X\right)^{-1}\right]$ and $D$ Optimal design satisfies the criterion $\mathrm{D}=\min \left|\left(\mathrm{X}^{\mathrm{T}} \mathrm{X}\right)^{-1}\right|$ or equivalently $\mathrm{D}=\max \left|\left(\mathrm{X}^{\mathrm{T}} \mathrm{X}\right)\right| \mathrm{A}$ design with minimum prediction variance $\min \left[N \hat{\sigma}_{\max }^{2}\right]$ is G-Optimal. Conversely, an efficient design is a design with high efficiency values. . When one has various designs at one's disposal, the most efficient design to choose is the one with larger efficiency values with smaller design size. The overall results for the twenty seven number combinations of different replications of the various portions of all the CCDs under study show that the efficiency of design is dependent on the optimality values; optimality values tend to influence the efficiency of design either negatively or positively. Larger optimality values depreciate the efficiency of the design while 
smaller optimality values improve efficiency of the design. The results, however, show that all the points where A, D and $G$ are optimal with maximum efficiency values were all partially replicated except for FCCD with $\mathrm{k}=5$ where the various portions were held fixed. Replicating a complete SCCD, RCCD, OCCD and FCCD with one $r_{f}, r_{\alpha}$ and $n_{c}$ r-times, yielded the same A, D and G-efficiency values. Generally, the best G-efficiencies for the various CCDs recorded overall superior performance of $85 \%$ and above efficiency values. The best D-efficiency recorded above $70 \%$ for SCCD and RCCD and below 70\% for OCCD and FCCD. The RCCD recorded the best A-efficiency value of $75.14 \%$. Super D-efficiency values of $104.03 \%$, $103.68 \%$ and $103.27 \%$ were also recorded by RCCD for factor $\mathrm{k}=5$ and $103.82 \%, 103.52 \%$, $103.18 \%, 107.64 \%, 107.30 \%, 107.0 \%, 100.30 \%, 100.20 \%$ and $100.20 \%$ for factor $\mathrm{k}=6$. To obtain optimal values and to have a good and efficient design, there is a need to replicate the cube, the axial and the center points. Partial replication of the various portions of the CCD tends to yield better results than equal replications. The best designs in terms of optimal and efficiency values seem to put more emphasis on replication of center point for SCCD, RCCD and OCCD but places more emphasis on replication of the axial point for FCCD.

\section{REFERENCES}

Boonorm, C. and Borkowski, J. J., (2012). "Comparison of response surface designs in a spherical region." International Journal of Mathematical and Computational Sciences, vol. 6, pp. 545-548.

Box, G. E. P. and Wilson, K. B. (1951). On the experimental attainment of optimum conditions. Journal of Royal Statistical Society, 13(1): 1-45.

Chernoff, H. (1953). Locally optimal designs for estimating parameters, Annals of Mathematical Statistics, 24, 586-602.

Chigbu, P. E. and Ohaegbulem, E. U. (2011). On the preference of replicating factorial runs in restricted second- order designs. Journal of Applied Sciences, 11(22): 3732-37.

Chigbu, P. E. and Ukaegbu, E. C. (2017). Recent developments on partial replications of response surface central composite designs: A review. An International Journal of Statistics Applications and Probability, 6(1): 91.

Crosier, R. B., (1993). Response surface design comparisons, Technical Report. U. S. army Edgewood research. Development and Engineering Center, SCBRD-RTM, Bldg. E3160 Aberdeen proving ground, Maryland, MD 21010-542.

Francis, C. E. \& Lilian, N. O. (2018). 'Alphabetic optimality criteria for $2^{K}$ central composite design'. Academic Journal of Applied Mathematical Sciences, 4, 107-118. http://arpgweb.com/?ic=journal\&journal=17\&info=aims

Ibanga, K. D. (2013). On optimal complete replicated rotatable, Orthogonal, Efficient and relative efficient central composite designs. Research Thesis.

Iwundu, M. P. (2015). Optimal partially replicated cube, star and center runs in face-central composite designs. International Journal of Statistics and Probability, 4(4): 1-19.

Iwundu, M. P., 2017. "The effects of addition of center points on the optimality of BoxBenhken and Box-Wilson Second-Order Designs." International Journal of Probability and Statistics, vol. 6, pp. 20-32.

Khuri, A. I. and Cornell, J. A., 1996. Response surfaces, Designs and analyses. 2nd ed. Marcel Dekker, Inc.

Myers, R.H., Khuri. A.I. \& Center W.H., Jr. (1966-1988). Response surface methodology. 
Nduka, U. C. \& Chigbu, P. E. (2014). "On optimal choice of cube and star replication in restricted second order designs communication in statistics-theory and methods". Journal Communications in Statistics Theory and Methods, 43, 4195-4214.

V. V Fedorov, Theory of Optimal Experiments, Academic Press, New York, 1972

Wald, A., (1943)."On the efficient design of statistical investigations." Ann. Math. Statist., $14,134-140$. 